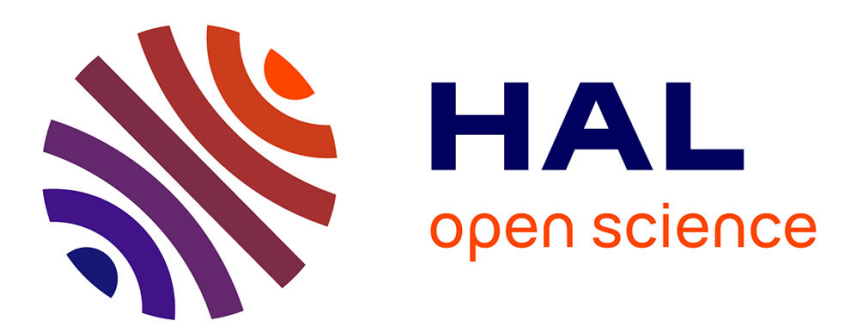

\title{
Implementation of Hybridizable Discontinuous Galerkin method for time-harmonic anisotropic poroelasticity in two dimensions.
}

Hélène Barucq, Julien Diaz, Rose-Cloé Meyer, Ha Pham

\section{- To cite this version:}

Hélène Barucq, Julien Diaz, Rose-Cloé Meyer, Ha Pham. Implementation of Hybridizable Discontinuous Galerkin method for time-harmonic anisotropic poroelasticity in two dimensions.. International Journal for Numerical Methods in Engineering, 2021, 10.1002/nme.6651 . hal-03464413

\author{
HAL Id: hal-03464413 \\ https://hal.inria.fr/hal-03464413
}

Submitted on 3 Dec 2021

HAL is a multi-disciplinary open access archive for the deposit and dissemination of scientific research documents, whether they are published or not. The documents may come from teaching and research institutions in France or abroad, or from public or private research centers.
L'archive ouverte pluridisciplinaire HAL, est destinée au dépôt et à la diffusion de documents scientifiques de niveau recherche, publiés ou non, émanant des établissements d'enseignement et de recherche français ou étrangers, des laboratoires publics ou privés. 
DOI: $\mathrm{xxx} / \mathrm{xxxx}$

\title{
Implementation of Hybridizable Discontinuous Galerkin method for time-harmonic anisotropic poroelasticity in two dimensions.
}

\author{
Hélène Barucq ${ }^{1,2}$ | Julien Diaz ${ }^{1,2}$ | Rose-Cloé Meyer*1,2 | Ha Pham ${ }^{1,2}$
}

${ }^{1}$ Project-team Magique-3D, INRIA Research Center Bordeaux Sud-Ouest, France

${ }^{2}$ LMAP, UPPA-E2S, Pau, France

\section{Correspondence}

*Rose-Cloé Meyer, Inria Magique 3D, UFR Sciences, Bât B1. Avenue de l'Université, F64013 Pau cedex. Email:

rose-cloe.meyer@inria.fr

\begin{abstract}
We apply a Hybridizable Discontinuous Galerkin (HDG) method to numerically solve two-dimensional anisotropic poroelastic wave equations in the frequency domain given by Biot theory. The motivation for choosing HDG method comes from the complexity of the considered equations and the high number of unknowns. The HDG method possesses all the advantages of Discontinuous Galerkin method (hpadaptivity, accuracy, ability to model complex tectonics,...) without a drastic increase in the number of degrees of freedom. After a description of its implementation, we illustrate the accuracy of the proposed method by comparisons with analytical solutions. We then perform a sensitivity analysis of the method as a function of stabilization parameters and frequency, and show in particular that there exists optimal values of these parameters in order to obtain a very good level of accuracy. We also show the ability of the method to reproduce the different types of poroelastic waves including the slow Biot wave.
\end{abstract}

\section{KEYWORDS:}

anisotropic poroelasticity, harmonic domain, Hybridizable Discontinuous Galerkin method

\section{1 | INTRODUCTION}

The simulation of wave propagation in poroelastic media is a significant step forward in the understanding and characterization of natural geological reservoirs which recieves a great interest with recent applications such as $\mathrm{CO} 2$ sequestration monitoring purposes ${ }^{1}$. Waves in poroelastic materials are governed by Biot's equations which characterize a geophysical material as consisting of an elastic solid frame and pores filled with fluid, air or gas. This physical model was introduced in a seminal work by Biot ${ }^{233}$ during the 60 s and has been used in many geophysical studies (see ${ }^{4 / 5}$ and their references therein for more discussion on the history). Compared to the acoustic or elastic model which were used for a long time for obvious computational reasons, Biot's equations employ a much larger number of physical parameters with some frequency-dependent, and thus provide a more accurate representation of geophysical layers by taking into consideration physical phenomena such as porosity or attenuation. Biot equations distinguish themselves from elastic wave equations by their capability to model three different types of waves. While an isotropic elastic medium sustains the well-known $\mathrm{P}$ and $\mathrm{S}$ waves, Biot model also includes the existence of an additional wave characteristics to isotropic poroelasticity called the Biot wave which propagates slower than $\mathrm{P}$ and $\mathrm{S}$ waves.

In terms of numerical simulations, grid-based numerical methods have long demonstrated a high potential for solving wave equations in complex media that may contain many heterogeneities of widely varying sizes. Works on porous media have employed from Finite Volume Method ${ }^{6}$, Boundary Element Method ${ }^{7}$, Finite Differences ${ }^{\frac{8}{8}}$, continuous Finite Element $\operatorname{Method}^{9}$, and Spectral-element methods (SEM) 1011 , a large majority of which being in the time domain. In particular, SEM demonstrated 
a clear efficiency in the time-domain, when based on hexahedral meshes. However, the representation of geological media is eased by using tetrahedral grids which are capable of more easily reproducing the geometric and constitutive variations of the medium. This is a clear motivation to turn to finite element methods authorizing tetrahedral meshes. Among them, Discontinuous Galerkin (DG) methods $\frac{12}{12}$, initially developed to solve fluid mechanics problems, have also been applied to wave propagation simulations in heterogeneous media, both in time domain ${ }^{13 / 14|15| 16}$ and frequency domain ${ }^{17 \mid 18}$.

The DG methods have many advantages like good performance on unstructured and irregular meshes thanks to $h p$ adaptivity. More importantly, each DG element communicates only with its direct neighbours. On a given mesh, the global matrix has thus a block structure, each block representing the interaction between two elements. This property is also a good point in favor of using high-order approximations since increasing the order does not increase the number of blocks of the matrix and does not modify the connectivity graph between these blocks. In addition, the DG block structure makes the method easily parallelizable. However, using discontinuous basis functions results in a significant increase in the number of degrees of freedom, such that the size of the overall discrete system is much larger than that of the system associated with a continuous finite element method. This is a real disadvantage when working in the frequency domain as one can easily reach the limits of direct solvers available in open-source that are essential to solve the problem 1

Hence, given that there is an obvious need in reducing the size of the linear system to be solved, we propose to consider a variant of DG methods which are called hybridizable. They are DG methods amenable to hybridization in the same sense as static condensation 19/20/21/22. Such methods require applying a local projection at the element level in order to parameterize the solution in terms of numerical traces. Then transmission conditions are constructed to connect the original solution to numerical traces. The original solution is then reconstructed a posteriori for negligible computational costs, cell by cell by solving local small-sized problems. Hybridizable DG (HDG) methods have been introduced in two seminal papers ${ }^{23 / 24}$ and successfully developed for many problems, as for example in ${ }^{25266}$ for acoustics and elastodynamics in ${ }^{2728}$ for electromagnetism, in ${ }^{2129130 \mid 31}$ for time-harmonic seismic waves. Here we limit the citations only to wave problems. The HDG solution methodology relies on the possibility of relating local unknowns at the element level to a so-called hybrid variable defined only of the skeleton of the mesh (i.e. the set of edges of triangles in 2D) by the mean of transmission conditions. In this way, the HDG solution is obtained by solving a smaller global system for the hybrid variable and the solution is reconstructed thanks to the solution of local systems in parallel.

As far as Biot consolidation model is concerned, the literature contains a lot of work on the finite element approximation

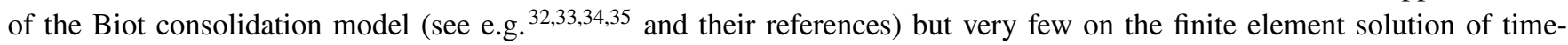
harmonic Biot equations. In two very recent works, $\mathrm{Fu}^{\sqrt{36}}$ and Hungria ${ }^{37}$ implement the HDG solution of poroelastic wave equations. $\mathrm{Fu}^{36}$ considers the quasi-static Biot equations using a displacement-pressure formulation. Hungria ${ }^{37}$ deals with both the time-dependent and time-harmonic Biot equations using a displacement-stress-pressure formulation. Herein, we consider a formulation of Biot equations governing the frame velocity, the relative fluid velocity, the pressure field, and the solid stress tensor, written in the frequency domain. Our approach is closer to that of Hungria since we consider time-harmonic equations whereas Fu solves quasi-static equations. But it is worth noting that we do not solve the same formulation as Hungria. As an advantage, the first order formulation gives us access to velocities and stress tensor which are the actual data displayed by experimental acquisition. This is very important if we want to use the code in an inversion loop for characterizing the propagation in the medium.

The paper is organized as follows. First, we briefly introduce in Section 2 the poroelastic wave equations together with the different physical parameters. Then, we present the HDG formulation in Section 3 and the associated discretization in Section 4. The description of the HDG solution methodology is accompanied by a numerical analysis aiming at ensuring the discrete problem is well-posed in Section 5 We carry out analysis of convergence and the conditioning of the system, see Section 5.2.3. We also provide some numerical experiments to validate the method 5 by comparing with analytical solutions that were developed in another of our work $\frac{38}{38}$. We finally give an example of 3-layer material in which we can simulate poroelastic effects and the three types of waves including the slow Biot wave.

\footnotetext{
${ }^{1}$ We can mitigate the large size of the linear system by using an iterative solver. However, with an application in iterative inversion in mind, we are more interested in a direct solver due to its multi right-hand-side features.
} 


\section{2 | EQUATIONS OF POROELASTICTY}

In this section, following Biot's model ${ }^{3}$, we present the two-dimensional anisotropic poroelastic wave equation set in the frequency domain. The system of equations will be given in first-order formulation to be solved with HDG method. We denote by $\omega$ the angular frequency which appears when restricting the study to time-harmonic waves having their time dependency as $e^{\mathrm{i} \omega t}$.

\section{1 | First-order harmonic equations}

A poroelastic medium is composed of a solid frame and pores filled with a fluid, and is characterized by the following physical parameters.

1. The porosity of the material $\phi$ is defined as $\phi=\frac{V_{f}}{V_{T}}$, with $V_{f}$ the fluid volume in the material and $V_{T}$ the total volume.

2. The averaged density $\rho_{a}$ is defined as a barycentric combination of the solid density $\rho_{s}$ and the fluid density $\rho_{f}, \rho_{a}=$ $(1-\phi) \rho_{s}+\phi \rho_{f}$, all in $\mathrm{kg} \mathrm{m}^{-3}$.

3. The dynamic density $\rho_{\text {dyn }}(\omega)$ given in $\mathrm{kg} \mathrm{m}^{-3}$ is defined as a function of the dynamic viscosity of the fluid $\eta$ (in Pa s) $\left(\mathrm{see}^{4} \mathrm{p} .25\right)$ and the dynamic permeability $k\left(\right.$ in $\left.\mathrm{m}^{2}\right)$ :

$$
\rho_{\text {dyn }}(\omega)=\frac{\mathrm{i} \eta}{\omega k(\omega)} \quad \text { with } \quad \frac{1}{k(\omega)}=\frac{1}{k_{0}}\left(\sqrt{1-\mathrm{i} \frac{4}{m} \frac{\omega}{\omega_{t}}}-\mathrm{i} \frac{\omega}{\omega_{t}}\right) \quad \text { and } \quad \omega_{t}:=\frac{\phi}{\mathrm{t} k_{0}} \frac{\eta}{\rho_{f}} .
$$

Here, the (dimensionless) number $m$ is taken equal to 8 and $\omega_{t}$ is the transition frequency with $t$ the tortuosity and $k_{0}$ the permeability. The frequency dependency of $\rho_{\mathrm{dyn}}$ implies that the velocities are frequency-dependent. In the above expression, we have denoted by $\sqrt{\bullet}$ the square root branch that uses the principal value, i.e. for $z \in \mathbb{C} \backslash\{0\}$,

$$
\sqrt{z}:=\sqrt{|z|} e^{\mathrm{i} \frac{\operatorname{Arg}(z)}{2}} \quad, \quad \operatorname{Arg}(z) \in(-\pi, \pi] .
$$

4. $\mathbf{C}_{\mathrm{fr}}$ is the stiffness tensor with component $C_{i j k l}$ for $i, j, k, l=1,2,3$, that reduces in Voigt notation, (cf $\left.{ }^{39} \mathrm{p} 13\right), \boldsymbol{\alpha}$ is the effective-stress matrix, and $M$ is the fluid-solid coupling modulus, with all three given in Pa. In the general case, $\boldsymbol{\alpha}$ and $M$ are given in ${ }^{39}$ [eqs. 7.137-7.140]. In the case of isotropic medium, $\mathbf{C}_{\mathrm{fr}}$ is described by the frame bulk modulus $\mathrm{k}_{\mathrm{fr}}$ and $\mu_{\mathrm{fr}}$ the frame shear modulus:

$$
C_{i j k l}=\left(\mathrm{k}_{\mathrm{fr}}-\frac{2}{3} \mu_{\mathrm{fr}}\right) \delta_{i j} \delta_{k l}+\mu_{\mathrm{fr}}\left(\delta_{i k} \delta_{j l}+\delta_{i l} \delta_{j k}\right),
$$

and the effective stress matrix reduces to $\alpha=\alpha \rrbracket$ where $\alpha$ also called the effective-stress coefficient is given by

$$
\alpha=1-\frac{\mathrm{k}_{\mathrm{fr}}}{\mathrm{k}_{\mathrm{s}}}, \quad \text { and } \quad \frac{1}{M}=\frac{\alpha}{\mathrm{k}_{\mathrm{s}}}+\phi\left(\frac{1}{\mathrm{k}_{\mathrm{f}}}-\frac{1}{\mathrm{k}_{\mathrm{s}}}\right),
$$

with $\mathrm{k}_{\mathrm{s}}, \mathrm{k}_{\mathrm{f}}$, and $\mathrm{k}_{\mathrm{fr}}$ respectively the solid, fluid and frame bulk modulus.

In first-order formulation, the poroelastic unknowns consist of $\mathbf{u}$ the frame velocity, and $\mathbf{w}$ the relative fluid velocity both of size 2, p the fluid pressure and $\tau$ the solid stress tensor of size $2 \times 2$. Let $\mathcal{D}$ be a physical domain in $\mathbb{R}^{2}$ with boundaries $\Gamma$. On the domain $\mathcal{D}$, the unknowns $\left(\mathbf{u}, \mathbf{w}, \boldsymbol{\tau}, \mathrm{p}\right.$ ) solve the following first-order system with external forces $\mathbf{f}_{u}$ and $\mathbf{f}_{w}$ ( cf Section 3.4 of $\left(\frac{38}{)}\right.$,

$$
\begin{cases}\mathrm{i} \omega \rho_{a} \mathbf{u}+\mathrm{i} \omega \rho_{f} \mathbf{w} & =\nabla \cdot \boldsymbol{\tau}+\mathbf{f}_{u}, \\ \mathrm{i} \omega \rho_{f} \mathbf{u}+\mathrm{i} \omega \rho_{\mathrm{dyn}}(\omega) \mathbf{w} & =-\nabla \mathrm{p}+\mathbf{f}_{w}, \\ \mathrm{i} \omega \boldsymbol{\tau}+\mathrm{i} \omega \boldsymbol{\alpha} \mathrm{p} & =\mathbf{C}_{\mathrm{fr}} \boldsymbol{\epsilon}(\mathbf{u}), \\ \mathrm{i} \omega \mathrm{p} & =-M \nabla \cdot \mathbf{w}-M \boldsymbol{\alpha}: \boldsymbol{\epsilon}(\mathbf{u}) .\end{cases}
$$

The first two equations are equations of motion, and the last two ones are constitutive laws. We have written the velocity strain tensor $\boldsymbol{\epsilon}=\frac{\nabla \mathbf{u}+(\nabla \mathbf{u})^{t}}{2}$, and the operation : denotes the tensor scalar product, i.e. for tensors $\boldsymbol{a}$ and $\boldsymbol{b}, \boldsymbol{a}: \boldsymbol{b}=\sum_{i, j} a_{i j} b_{i j}$. 


\section{2 | Nondimensionalization of the equations}

For numerical implementation, the parameters may have different orders of magnitude. To manipulate numbers with a similar order of magnitude, we write the system (1) with no source in terms of dimensionless equations. We write $X=X_{0} \bar{X}$ for all the terms of the equations with $X_{0}$ a characteristic unit of measure and $\bar{X}$ denoting non-dimensional quantities. We assume that the components of $\mathbf{C}$ are written as $C_{0} \overline{\mathbf{C}}$, and that the bulk moduli k. are written as k. $=C_{0} \overline{\mathrm{k}}$. for $\bullet=\mathrm{s}$, f, fr. Hence, $M=C_{0} \bar{M}$ and $\alpha_{0}=1$. The porosity $\phi=\frac{V_{f}}{V_{T}}$ is considered as a ratio with no characteristic unit. We assume that $\rho_{a}, \rho_{\mathrm{dyn}}, \rho_{f}$ and $\rho_{s}$ have the same characteristic unit $\rho_{0}$. The characteristic frequency has the same characteristic unit as $\omega$. The system (1) with physical parameters becomes:

$$
\left\{\begin{array}{l}
\mathrm{i} \omega_{0} \bar{\omega} \rho_{0} \overline{\rho_{a}} \mathrm{u}_{0} \overline{\mathbf{u}}+\mathrm{i} \omega_{0} \bar{\omega} \rho_{0} \overline{\rho_{f}} \mathrm{w}_{0} \overline{\mathbf{w}}=\frac{\tau_{0}}{x_{0}} \nabla \cdot \overline{\boldsymbol{\tau}}, \\
\mathrm{i} \omega_{0} \bar{\omega} \rho_{0} \overline{\rho_{f}} \mathrm{u}_{0} \overline{\mathbf{u}}+\mathrm{i} \omega_{0} \bar{\omega} \rho_{0} \overline{\rho_{\mathrm{dyn}}} \mathrm{w}_{0} \overline{\mathbf{w}}=-\frac{\mathrm{p}_{0}}{x_{0}} \nabla \overline{\mathrm{p}}, \\
\mathrm{i} \omega_{0} \bar{\omega} \tau_{0} \overline{\boldsymbol{\tau}}+\mathrm{i} \omega_{0} \bar{\omega} \alpha_{0} \overline{\boldsymbol{\alpha}} \mathrm{p}_{0} \overline{\mathrm{p}} \rrbracket=\frac{C_{0} \mathbf{u}_{0}}{x_{0}} \overline{\mathbf{C}} \boldsymbol{\epsilon}(\overline{\mathbf{u}}), \\
\mathrm{i} \omega_{0} \bar{\omega} \mathrm{p}_{0} \overline{\mathrm{p}}=-\frac{C_{0} \mathrm{w}_{0}}{x_{0}} \bar{M} \nabla \cdot \overline{\mathbf{w}}-\frac{C_{0} \alpha_{0} \mathrm{u}_{0}}{x_{0}} \bar{M} \overline{\boldsymbol{\alpha}}: \epsilon(\overline{\mathbf{u}}), \\
\rho_{0} \overline{\rho_{\mathrm{dyn}}}=\frac{\eta_{0}}{\kappa_{0_{0}} \omega_{0}} \frac{\bar{\eta}}{\bar{k}} .
\end{array}\right.
$$

The equations are written with dimensionless quantities, and all the constants must be equal. Simplifying, we have:

$$
\mathrm{u}_{0}=\mathrm{w}_{0}, \quad \tau_{0}=\mathrm{p}_{0}=\omega_{0} \rho_{0} \mathrm{u}_{0} x_{0}, \quad C_{0} u_{0}=\omega_{0} \tau_{0} x_{0}, \quad \eta_{0}=\rho_{0} \omega_{0} k_{0_{0}} .
$$

In many geophysical materials, the magnitude of the bulk modulus and the stiffness tensor is GPa or MPa, and the magnitude of the densities is in $10^{3} \mathrm{~kg} \cdot \mathrm{m}^{-3}$. We hence choose:

$$
C_{0}=10^{9} \mathrm{~Pa}, \quad \rho_{0}=10^{3} \mathrm{~kg} \cdot \mathrm{m}^{-3},
$$

and this follows,

$$
\tau_{0}=\mathrm{p}_{0}=10^{6} \mathrm{~Pa}, \quad \omega_{0}=10^{3} \mathrm{rad} . \mathrm{s}^{-1}, \quad x_{0}=1 \mathrm{~m}, \quad \mathrm{u}_{0}=\mathrm{w}_{0}=1 \mathrm{~m} . \mathrm{s}^{-1}, \quad \eta_{0}=10^{-3} \text { Pa.s }, \quad \kappa_{0_{0}}=10^{-9} \mathrm{~m}^{2} .
$$

We note that in other applications using different materials, the magnitude of the physical parameters can differ, and the results presented above can be no longer relevant. In the rest of the paper, the overbars denoting the non-dimensionalized quantities are dropped.

\section{3 | Boundary conditions}

On the external boundary $\Gamma$ with outwardly direct unit normal vector $\boldsymbol{n}$, we consider four types of boundary conditions with vector $f_{\text {inc }}$ and scalar $g_{\text {inc }}$ denoting the exterior boundary forces,

$$
\begin{aligned}
& \text { Type } 1 \quad\left\{\begin{array} { r l } 
{ \boldsymbol { \tau } \boldsymbol { n } } & { = \boldsymbol { f } _ { \text { inc } } , } \\
{ \mathbf { w } \cdot \boldsymbol { n } } & { = g _ { \text { inc } } , }
\end{array} \quad \text { (4a) } \quad \text { Type } 2 \quad \left\{\begin{array}{rl}
\boldsymbol{\tau} \boldsymbol{n} & =\boldsymbol{f}_{\text {inc }}, \\
\mathrm{p} & =g_{\text {inc }},
\end{array}\right.\right. \\
& \text { Type } 3 \quad\left\{\begin{array} { l } 
{ \mathbf { u } = \boldsymbol { f } _ { \text { inc } } , } \\
{ \mathrm { p } = g _ { \text { inc } } , }
\end{array} \quad \text { (4c) Type } 4 \quad \left\{\begin{array}{c}
\mathbf{u}=\boldsymbol{f}_{\text {inc }}, \\
\mathbf{w} \cdot \boldsymbol{n}=g_{\text {inc }} .
\end{array}\right.\right.
\end{aligned}
$$

It is worth noting that the conditions $4 \mathrm{~b}$ ) include the free boundary conditions which are given by

$$
\boldsymbol{\tau} \boldsymbol{n}=\mathbf{0}, \mathrm{p}=0, \quad \text { Free surface boundary condition }
$$


To alleviate the notation, we write equation (1) with one of the boundary conditions in (4) as

$$
\begin{aligned}
\mathcal{L}_{\text {poro }} \mathcal{U} & =\left(\boldsymbol{f}_{\text {vol }}, \boldsymbol{g}_{\text {vol }}, \mathbf{0}, 0\right)^{T} & \text { in } \mathcal{D}, \\
\mathcal{T}_{i} \mathfrak{U} & =\left(\boldsymbol{f}_{\text {inc }}, g_{\text {inc }}\right)^{T} & \text { on } \partial \mathcal{D} .
\end{aligned}
$$

Here, we have introduced the notation of the poroelastic operator $\mathcal{L}_{\text {poro }}$, and boundary operator $\mathcal{T}_{i}$ for $\mathrm{i}=1,4$. In this notation, the unknown is $\mathcal{U}=(\mathbf{u}, \mathbf{w}, \boldsymbol{\tau}, \mathrm{p})^{T}$, and $\mathbf{0}$ in the volume right-hand-side is the zero (2nd-order) tensor. The right-hand-side in the boundary conditions consists of the vector-valued function $f_{\text {inc }}$ and the scalar function $g_{\text {inc }}$. In the following, we consider boundary conditions of type 1 . We present in Appendix B how to handle the presence of the different boundary types.

\section{3 | FORMULATION OF HDG METHOD}

The poroelastic equations are complex and need a lot of computational power, because of the size of the system. We choose to use HDG method, to get high-order results. The method is based on DG methods, and presents the same advantages to be usable on unstructured meshes, with discontinuous basis functions. This is also a method on which we can use hp-adaptivity and that is easily parallelizable because the calculations can be done elementwise. The main drawback of DG methods is the large number of degrees of freedom compared to finite element methods, which increases the computational cost. In HDG methods, the unknowns are expressed as functions of a hybrid unknown, only defined on the boundaries of the elements ${ }^{40}$. The hybrid unknown is taken as a Lagrange multiplier which satisfies a global system defined from the surface degrees of freedom attached to the boundaries of the elements. This reduces the number of degrees of freedom ${ }^{29}$. In this section, we apply the HDG method to the poroelastic wave equations.

\section{1 | Notations}

On a domain $\mathcal{D}$, we will work with the following function spaces,

$$
\begin{aligned}
& L^{2}(\mathcal{D}), \text { the space of square-integrable functions on the domain } \mathcal{D}, \\
& V^{p}(\mathcal{D}), \text { the set of polynomials of degree at most } p \text { on } \mathcal{D}, \\
& V^{p}(\mathcal{D})=\left(V^{p}(\mathcal{D})\right)^{2} \\
& \boldsymbol{\Sigma}^{p}(\mathcal{D})=\left\{\tau \in\left(V^{p}(\mathcal{D})\right)^{4} \mid \tau_{y x}=\tau_{x y}\right\}
\end{aligned}
$$

We next consider a triangulation $\mathcal{T}_{h}$ of the domain $\mathcal{D}$ of dimension 2. $K$ denotes an element of the mesh, which in 2D is a triangle. We denote by $\boldsymbol{F}$ a face of the element $K$, and $\boldsymbol{n}$ the unit normal vector to $F$. Associated to triangulation $\mathcal{T}_{h}$, we define the following spaces:

$$
\begin{aligned}
V_{h}^{p} & =\left\{v \in L^{2}(\mathcal{D}):\left.v\right|_{K} \in V^{p}(K), \forall K \in \mathcal{T}_{h}\right\}, \\
\boldsymbol{V}_{h}^{p} & =\left\{\boldsymbol{v} \in\left(L^{2}(\mathcal{D})\right)^{2}:\left.\boldsymbol{v}\right|_{K} \in V^{p}(K), \forall K \in \mathcal{T}_{h}\right\}, \\
\boldsymbol{\Sigma}_{h}^{p} & =\left\{\tau \in L^{2}(\mathcal{D})^{4}:\left.\tau\right|_{K} \in \boldsymbol{\Sigma}^{p}(K), \forall K \in \mathcal{T}_{h}\right\}, \\
M_{h} & =\left\{\xi \in L^{2}\left(\mathcal{F}_{h}\right):\left.\xi\right|_{F} \in V^{p}(F),, \forall K \in \mathcal{F}_{h}\right\}, \\
M_{h} & =\left\{\eta \in\left(L^{2}\left(\mathcal{F}_{h}\right)\right)^{2}:\left.\eta\right|_{F} \in\left(V^{p}(F)\right)^{2}, \forall K \in \mathcal{F}_{h}\right\} .
\end{aligned}
$$

Remark 1. Note that we impose strongly the symmetry on the stress tensor $\tau$, which prevents the potential loss of half an order described in ${ }^{41}$ and $\mathrm{d}^{42}$.

The jumps $\llbracket \cdot \rrbracket$ are defined on an interior face $F=\partial K \cap \partial K^{\prime}$ as

$$
\llbracket \mathbf{w} \cdot n \rrbracket=\mathbf{w}^{K} \cdot n^{K}+\mathbf{w}^{K^{\prime}} \cdot n^{K^{\prime}}, \quad \llbracket \tau n \rrbracket=\tau^{K} n^{K}+\tau^{K^{\prime}} n^{K^{\prime}}
$$

and on a boundary face,

$$
\llbracket \mathbf{w} \cdot n \rrbracket=\mathbf{w}^{K} \cdot n^{K}, \quad \llbracket \tau n \rrbracket=\tau^{K} n^{K}
$$




\section{2 | Local problem}

We consider an element $K$ of $\mathcal{T}_{h}$, and an exact variational solution of (1) on $K$ denoted by (u, w, $\tau$, p). Define the following test functions: $(\tilde{\mathbf{u}}, \tilde{\mathbf{w}}, \tilde{\boldsymbol{\tau}}, \tilde{\mathrm{p}}) \in\left(\boldsymbol{V}^{p}(K) \times \boldsymbol{V}^{p}(K) \times \boldsymbol{\Sigma}^{p}(K) \times V^{p}(K)\right)$. The integration on an element of (1) gives:

$$
\begin{gathered}
\int_{K} \mathrm{i} \omega \rho_{a} \mathbf{u} \cdot \tilde{\mathbf{u}}+\int_{K} \mathrm{i} \omega \rho_{f} \mathbf{w} \cdot \tilde{\mathbf{u}}-\int_{K}(\nabla \cdot \tau) \cdot \tilde{\mathbf{u}}=\int_{K} f_{\mathrm{vol}} \cdot \tilde{\mathbf{u}}, \\
\int_{K} \mathrm{i} \omega \rho_{f} \mathbf{u} \cdot \tilde{\mathbf{w}}+\int_{K} \mathrm{i} \omega \rho_{\mathrm{dyn}} \mathbf{w} \cdot \tilde{\mathbf{w}}+\int_{K}(\nabla \mathrm{p}) \cdot \tilde{\mathbf{w}}=\int_{K} \boldsymbol{g}_{\mathrm{vol}} \cdot \tilde{\mathbf{w}}, \\
\int_{K} \mathrm{i} \omega \boldsymbol{\tau}: \tilde{\boldsymbol{\tau}}+\int_{K} \mathrm{i} \omega \boldsymbol{\alpha} \mathrm{p}: \tilde{\boldsymbol{\tau}}-\int_{K}\left(\mathbf{C}_{\mathrm{fr}} \boldsymbol{\epsilon}(\mathbf{u})\right): \tilde{\boldsymbol{\tau}}=0, \\
\int_{K} \mathrm{i} \omega \mathrm{p} \tilde{\mathrm{p}}+\int_{K} M \nabla \cdot \mathbf{w} \tilde{\mathrm{p}}+\int_{K} M \boldsymbol{\alpha}: \boldsymbol{\epsilon}(\mathbf{u}) \tilde{\mathrm{p}}=0 .
\end{gathered}
$$

By integrating by parts, we have:

$$
\begin{gathered}
\int_{K} \mathrm{i} \omega \rho_{a} \mathbf{u} \cdot \tilde{\mathbf{u}}+\int_{K} \mathrm{i} \omega \rho_{f} \mathbf{w} \cdot \tilde{\mathbf{u}}+\int_{K} \boldsymbol{\tau}: \nabla \tilde{\mathbf{u}}-\int_{\partial K} \hat{\boldsymbol{\tau}} \boldsymbol{n} \cdot \tilde{\mathbf{u}}=\int_{K} \boldsymbol{f}_{\mathrm{vol}} \cdot \tilde{\mathbf{u}}, \\
\int_{K} \mathrm{i} \omega \rho_{f} \mathbf{u} \cdot \tilde{\mathbf{w}}+\int_{K} \mathrm{i} \omega \rho_{\mathrm{dyn}} \mathbf{w} \cdot \tilde{\mathbf{w}}-\int_{K} \mathrm{p} \nabla \cdot \tilde{\mathbf{w}}+\int_{\partial K} \hat{\mathrm{p}} \boldsymbol{n} \cdot \tilde{\mathbf{w}}=\int_{K} \boldsymbol{g}_{\mathrm{vol}} \cdot \tilde{\mathbf{w}}, \\
\int_{K} \mathrm{i} \omega \tau: \tilde{\boldsymbol{\tau}}+\int_{K} \mathrm{i} \omega \boldsymbol{\alpha p}: \tilde{\boldsymbol{\tau}}+\int_{K} \mathbf{u} \cdot \nabla \cdot\left(\mathbf{C}_{\mathrm{fr}} \tilde{\boldsymbol{\tau}}\right)-\int_{\partial K} \hat{\mathbf{u}} \cdot \mathbf{C}_{\mathrm{fr}} \tilde{\boldsymbol{\tau}} \boldsymbol{n}=0, \\
\int_{K} \mathrm{i} \omega \mathrm{p} \tilde{\mathrm{p}}-\int_{K} M \mathbf{w} \cdot \nabla \tilde{\mathrm{p}}+\int_{\partial K} M(\hat{\mathbf{w}} \cdot \boldsymbol{n}) \tilde{\mathrm{p}}-\int_{K} M(\boldsymbol{\alpha} \mathbf{u}) \cdot \nabla \tilde{\mathrm{p}}+\int_{\partial K} M(\boldsymbol{\alpha} \hat{\mathbf{u}}) \cdot \boldsymbol{n} \tilde{\mathrm{p}}=0 .
\end{gathered}
$$

In the above equation, $\int_{K}\left(\mathbf{C}_{\mathrm{fr}} \boldsymbol{\epsilon}\left(\mathbf{u}_{h}\right)\right): \tilde{\boldsymbol{\tau}}=\int_{K} \boldsymbol{\epsilon}\left(\mathbf{u}_{h}\right): \mathbf{C}_{\mathrm{fr}} \tilde{\boldsymbol{\tau}}$ because $\mathbf{C}_{\mathrm{fr}}$ is symmetric.

\section{Introduction of the trace variables:}

The exact solution ( $\mathbf{u}, \mathbf{w}, \boldsymbol{\tau}, \mathrm{p})$ on $K$ is approximated by $\left(\mathbf{u}_{h}, \mathbf{w}_{h}, \boldsymbol{\tau}_{h}, \mathrm{p}_{h}\right) \in\left(\boldsymbol{V}_{h}^{p} \times \boldsymbol{V}_{h}^{p} \times \boldsymbol{\Sigma}_{h}^{p} \times V_{h}^{p}\right)$. The traces on $\partial K$ are approximated by numerical traces $\left(\hat{\mathbf{u}}_{h}, \hat{\mathbf{w}}_{h} \cdot \boldsymbol{n}, \hat{\boldsymbol{\tau}}_{h} \boldsymbol{n}, \hat{\mathrm{p}}_{h}\right)$. The principle of HDG consists in choosing two traces, one among the "solid" group, $\hat{\boldsymbol{\tau}}_{h} \boldsymbol{n}$ and $\hat{\mathbf{u}}_{h}$, and one among the "fluid" group $\hat{\mathbf{w}}_{h} \cdot \boldsymbol{n}$ and $\hat{\mathrm{p}}_{h}$, and replacing them by auxiliary unknowns defined only on the edges of the element. This choice should ensure the well-posedness of the local problem when the auxiliary unknowns are used as boundary conditions. We recall that for the acoustic equation, the most common choice is $\hat{\mathrm{p}}_{h}{ }^{31}$, while for the elastic equation, $\hat{\mathbf{u}}_{h}{ }^{30}$ is chosen, even if choosing $\hat{\boldsymbol{\tau}}_{h} \boldsymbol{n}$ should be equivalent. For the poroelastic equations considered, there is no particular justification to prefer $\hat{\mathrm{p}}_{h}$ to $\hat{\mathbf{w}}_{h} \cdot \boldsymbol{n}$ in the fluid group since both traces are scalar, so that the size of the resulting global system is the same for both choices, and such a choice has no particular impact on the accuracy.

Following the common choice, we have arbitrarily chosen to define the two unknowns $\lambda_{1} \in \boldsymbol{M}_{h}, \lambda_{2} \in M_{h}$ to replace the numerical traces $\hat{\mathbf{u}}_{h}$ and $\hat{\mathrm{p}}_{h}$ :

$$
\lambda_{1}=\hat{\mathbf{u}}_{h}, \quad \lambda_{2}=\hat{\mathrm{p}}_{h}, \quad \forall F \in \mathcal{F}_{h} .
$$

If the choice of the two "main" traces is arbitrary, the definition of the "secondary" traces is crucial. Indeed, these numerical traces are defined as an approximation of the continuous traces with an additional penalization term that guarantees the order of convergence of the method. In acoustics, this penalization term is proportional to $\hat{\mathrm{p}}_{h}-\lambda^{\sqrt[31]{31}}$ while in elasticity, it is proportional to $\hat{\mathbf{u}}_{h}-\lambda^{30}$. Thus, the most natural choice for the considered poroelastic problem should be to penalize the trace of $\hat{\boldsymbol{\tau}}_{h} \boldsymbol{n}$ by a 
term proportional to $\hat{\mathbf{u}}_{h}-\lambda_{1}$ and the trace of $\hat{\mathbf{w}}_{h}$ by a term proportional to $\hat{\mathrm{p}}_{h}-\lambda_{2}$. However, since all the unknowns are coupled, we propose instead to define $\left(\hat{\boldsymbol{\tau}}_{h} \boldsymbol{n}, \hat{\mathbf{w}}_{h} \cdot \boldsymbol{n}\right)$ as

$$
\left\{\begin{array}{l}
\hat{\boldsymbol{\tau}}_{h} \boldsymbol{n}=\boldsymbol{\tau}_{h} \boldsymbol{n}-\boldsymbol{S}_{1}\left(\mathbf{u}_{h}-\lambda_{1}\right)-\left(\mathrm{p}_{h}-\lambda_{2}\right) \boldsymbol{S}_{3} \boldsymbol{n}, \\
\hat{\mathbf{w}}_{h} \cdot \boldsymbol{n}=\mathbf{w}_{h} \cdot \boldsymbol{n}-\left(\left(\mathrm{p}_{h}-\lambda_{2}\right) \boldsymbol{S}_{2} \boldsymbol{n}\right) \cdot \boldsymbol{n}-\boldsymbol{S}_{4}\left(\left(\mathbf{u}_{h}-\lambda_{1}\right)\right) \cdot \boldsymbol{n},
\end{array}\right.
$$

where $S_{1}, S_{2}, S_{3}, S_{4}$ are called the stabilization matrices, with $S_{1}$ representing the stabilization on the solid frame, $\boldsymbol{S}_{2}$ on the fluid, while $\boldsymbol{S}_{3}$ and $\boldsymbol{S}_{4}$ are associated with the coupling of these two materials that compose the porous medium.

Remark 1. Note that the fact that we use four penalization parameters instead of two, as in the works ${ }^{\sqrt{36}}$ and ${ }^{37}$, does not impact the computational cost of the methods. Indeed, this will only change the value of the coefficient of the global matrix, but not its profile. In section 5.2.2, we will analyze the influence of these four parameters on the accuracy of the scheme and we will show that they are all necessary to guarantee the order of convergence of our formulation.

The numerical traces are replaced in (12) to give:

$$
\begin{gathered}
\int_{K} \mathrm{i} \omega \rho_{a}^{K} \mathbf{u}_{h} \cdot \tilde{\mathbf{u}}+\int_{K} \mathrm{i} \omega \rho_{f}^{K} \mathbf{w}_{h} \cdot \tilde{\mathbf{u}}+\int_{K} \boldsymbol{\tau}_{h}: \nabla \tilde{\mathbf{u}}-\int_{\partial K} \hat{\boldsymbol{\tau}}_{h} \boldsymbol{n} \cdot \tilde{\mathbf{u}} \\
+\int_{\partial K} \boldsymbol{S}_{1}\left(\mathbf{u}_{h}-\lambda_{1}\right) \cdot \tilde{\mathbf{u}}+\int_{\partial K}\left(\mathrm{p}_{h}-\lambda_{2}\right)\left(\boldsymbol{S}_{3} \boldsymbol{n}\right) \cdot \tilde{\mathbf{u}}=\int_{K} f_{\mathrm{vol}}^{K} \cdot \tilde{\mathbf{u}}, \\
\int_{K} \mathrm{i} \omega \rho_{f}^{K} \mathbf{u}_{h} \cdot \tilde{\mathbf{w}}+\int_{K} \mathrm{i} \omega \rho_{\mathrm{dyn}}^{K} \mathbf{w}_{h} \cdot \tilde{\mathbf{w}}-\int_{K} \mathrm{p}_{h} \nabla \cdot \tilde{\mathbf{w}}+\int_{\partial K} \lambda_{2} \boldsymbol{n} \cdot \tilde{\mathbf{w}}=\int_{K} \boldsymbol{g}_{\mathrm{vol}}^{K} \cdot \tilde{\mathbf{w}}, \\
\int_{K} \mathrm{i} \omega \boldsymbol{\tau}_{h}: \tilde{\boldsymbol{\tau}}+\int_{K} \mathrm{i} \omega \boldsymbol{\alpha}^{K} \mathrm{p}_{h}: \tilde{\boldsymbol{\tau}}+\int_{K} \mathbf{u}_{h} \cdot \nabla \cdot\left(\mathbf{C}_{\mathrm{fr}}^{K} \tilde{\boldsymbol{\tau}}\right)-\int_{\partial K} \lambda_{1} \cdot \mathbf{C}_{\mathrm{fr}}^{K} \tilde{\boldsymbol{\tau}} \boldsymbol{n}=0 \\
\int_{K} \mathrm{i} \omega \mathrm{p}_{h} \tilde{\mathrm{p}}-\int_{K} M_{\mathbf{w}_{h}} \cdot \nabla \tilde{\mathrm{p}}+\int_{\partial K} M\left(\hat{\mathbf{w}}_{h} \cdot \boldsymbol{n}\right) \tilde{\mathrm{p}}-\int_{\partial K} M^{K}\left(\mathrm{p}_{h}-\lambda_{2}\right)\left(\boldsymbol{S}_{2} \boldsymbol{n}\right) \cdot \boldsymbol{n} \tilde{\mathrm{p}} \\
-\int_{\partial K} M^{K} \boldsymbol{S}_{4}\left(\mathbf{u}_{h}-\lambda_{1}\right) \cdot \boldsymbol{n} \cdot \tilde{\mathrm{p}}-\int_{K} M^{K}\left(\boldsymbol{\alpha}^{K} \mathbf{u}_{h}\right) \cdot \nabla \tilde{\mathrm{p}}+\int_{\partial K} M^{K}\left(\boldsymbol{\alpha}^{K} \lambda_{1}\right) \cdot \boldsymbol{n} \tilde{\mathrm{p}}=0 .
\end{gathered}
$$

By integrating by parts, we obtain the local problem:

$$
\begin{gathered}
\int_{K} \mathrm{i} \omega \rho_{a}^{K} \mathbf{u}_{h} \cdot \tilde{\mathbf{u}}+\int_{K} \mathrm{i} \omega \rho_{f}^{K} \mathbf{w}_{h} \cdot \tilde{\mathbf{u}}-\int_{K}\left(\nabla \cdot \boldsymbol{\tau}_{h}\right) \cdot \tilde{\mathbf{u}}+\int_{\partial K} \boldsymbol{S}_{1}\left(\mathbf{u}_{h}-\lambda_{1}\right) \cdot \tilde{\mathbf{u}}+\int_{\partial K}\left(\mathrm{p}_{h}-\lambda_{2}\right)\left(\boldsymbol{S}_{3} \boldsymbol{n}\right) \cdot \tilde{\mathbf{u}}=\int_{K} f_{\mathrm{vol}}^{K} \cdot \tilde{\mathbf{u}}, \\
\int_{K} \mathrm{i} \omega \rho_{f}^{K} \mathbf{u}_{h} \cdot \tilde{\mathbf{w}}+\int_{K} \mathrm{i} \omega \rho_{\mathrm{dyn}}^{K} \mathbf{w}_{h} \cdot \tilde{\mathbf{w}}-\int_{K} \mathrm{p}_{h} \nabla \cdot \tilde{\mathbf{w}}+\int_{\partial K} \lambda_{2} \boldsymbol{n} \cdot \tilde{\mathbf{w}}=\int_{K} \boldsymbol{g}_{\mathrm{vol}}^{K} \cdot \tilde{\mathbf{w}} \\
\int_{K} \mathrm{i} \omega \tau_{h}: \tilde{\boldsymbol{\tau}}+\int_{K} \mathrm{i} \omega \boldsymbol{\alpha} \mathrm{p}_{h}: \tilde{\boldsymbol{\tau}}+\int_{K} \mathbf{u}_{h} \cdot \nabla \cdot\left(\mathbf{C}_{\mathrm{fr}}^{K} \tilde{\boldsymbol{\tau}}\right)-\int_{\partial K} \lambda_{1} \cdot\left(\mathbf{C}_{\mathrm{fr}}^{K} \tilde{\boldsymbol{\tau}} \boldsymbol{n}\right)=0 \\
\int_{K} \mathrm{i} \omega \mathrm{p}_{h} \tilde{\mathrm{p}}+\int_{K} M \nabla \cdot \mathbf{w}_{h} \tilde{\mathrm{p}}-\int_{\partial K} M\left(\mathrm{p}_{h}-\lambda_{2}\right)\left(\boldsymbol{S}_{2} \boldsymbol{n}\right) \cdot \boldsymbol{n} \tilde{\mathrm{p}}-\int_{\partial K} M \boldsymbol{S}_{4}\left(\mathbf{u}_{h}-\lambda_{1}\right) \cdot \boldsymbol{n} \tilde{\mathrm{p}} \\
-\int_{K} M\left(\boldsymbol{\alpha} \cdot \mathbf{u}_{h}\right) \cdot \nabla \tilde{\mathrm{p}}+\int_{\partial K} M\left(\boldsymbol{\alpha} \lambda_{1}\right) \cdot \boldsymbol{n} \tilde{\mathrm{p}}=0 .
\end{gathered}
$$




\section{3 | Transmission conditions}

The HDG formulation is established by combining the local problem with two transmission conditions at the interfaces of the mesh. Let $(\eta, \xi) \in \boldsymbol{M}_{h} \times \boldsymbol{M}_{h}$ be two test-functions defined on the faces of the element $K$, and recall the jumps for an interior face (9), the transmission conditions are:

$$
\sum_{F \in \mathcal{F}_{h}} \int_{F} \llbracket \hat{\boldsymbol{\tau}}_{h} \boldsymbol{n} \rrbracket \cdot \eta=\sum_{F \in \mathcal{F}_{\mathrm{ext}}} \int_{F} \boldsymbol{f}_{\mathrm{inc}} \cdot \eta
$$

$$
\sum_{F \in \mathcal{F}_{h}} \int_{F} \llbracket \hat{\mathbf{w}}_{h} \cdot \boldsymbol{n} \rrbracket \xi=\sum_{F \in \mathcal{F}_{\text {ext }}} \int_{F} g_{\text {inc }} \xi
$$

\section{4 | DISCRETIZATION USING HDG METHOD}

The following section details the two-dimensional discretization of the HDG method in the $(x, y)$ plane. In general formulation of HDG 2 27/29/30/43, the stabilization matrices $\boldsymbol{S}$ are just required to be positive definite. However, in practice, they are mostly set to be a scalar times the identity matrices. From now, we assume that the stabilization matrices in the HDG formulation, cf. (14), are diagonal, i.e. $S_{i}=\gamma_{i}$ 『 for $i=1$, 4. In this way, the expression (14) simplifies to

$$
\left\{\begin{array}{l}
\hat{\boldsymbol{\tau}}_{h} \boldsymbol{n}=\boldsymbol{\tau}_{h} \boldsymbol{n}-\gamma_{1}\left(\mathbf{u}_{h}-\lambda_{1}\right)-\left(\mathrm{p}_{h}-\lambda_{2}\right) \gamma_{3} \boldsymbol{n}, \\
\hat{\mathbf{w}}_{h} \cdot \boldsymbol{n}=\mathbf{w}_{h} \cdot \boldsymbol{n}-\gamma_{2}\left(\mathrm{p}_{h}-\lambda_{2}\right)-\gamma_{4}\left(\mathbf{u}_{h}-\lambda_{1}\right) \cdot \boldsymbol{n} .
\end{array}\right.
$$

In the formulation, the test functions are decomposed in the basis functions of $V_{p}$ as $\varphi_{i}^{K}$ for $\tilde{\mathbf{u}}, \tilde{\mathbf{w}}, \tilde{\boldsymbol{\tau}}, \tilde{\mathrm{p}}$ and $\psi_{j}^{F}$ for $\eta, \xi$. The local solutions are expressed along $x$ and $y$ directions:

$$
\mathbf{u}_{h}=\left(\begin{array}{c}
\mathrm{u}_{x}^{K} \\
\mathrm{u}_{y}^{K}
\end{array}\right), \quad \mathbf{w}_{h}=\left(\begin{array}{c}
\mathrm{w}_{x}^{K} \\
\mathrm{w}_{y}^{K}
\end{array}\right), \quad \tau_{h}=\left(\begin{array}{cc}
\tau_{x x}^{K} & \tau_{x y}^{K} \\
\tau_{x y}^{K} & \tau_{y y}^{K}
\end{array}\right) .
$$

They are decomposed as:

$$
\mathrm{u}_{l}^{K}=\sum_{j=1}^{d_{i}^{K}} \underline{\mathrm{u}}_{l, j}^{K} \varphi_{j}^{K}, \quad \mathrm{w}_{l}^{K}=\sum_{j=1}^{d_{i}^{K}} \underline{\mathrm{w}}_{l, j}^{K} \varphi_{j}^{K}, \quad \tau_{k l}^{K}=\sum_{j=1}^{d_{i}^{K}} \underline{\tau}_{k l, j}^{K} \varphi_{j}^{K}, \quad \mathrm{p}_{h}^{K}=\sum_{j=1}^{d_{i}^{K}} \underline{\mathrm{p}}^{K} \varphi_{j}^{K},
$$

where $d_{i}^{K}$ denotes the number of degrees of freedom of an element, and with $l=x, y$ and $k=x, y$. The local Lagrange unknowns are decomposed as follows:

$$
\lambda_{1 l}^{F}=\sum_{j=1}^{d_{i}^{F}} \underline{\lambda}_{1 l}^{F} \psi_{j}^{F}, \quad \lambda_{2}^{F}=\sum_{j=1}^{d_{i}^{F}} \underline{\lambda}_{j}^{F} \psi_{j}^{F}, \quad \text { with } l=x, y
$$

where $d_{i}^{F}$ denotes the number of degrees of freedom of an edge. In (20), each component is considered as a vector of the form $\underline{u}_{l}^{K}=\left(\underline{u}_{l, 1}^{K}, \ldots, \underline{u}_{, d_{i}^{K}}^{K}\right)^{T}$ for $l=x, y$. Similarly, every local component of the unknowns in (21) is written as: $\lambda_{1 l}^{F}=$

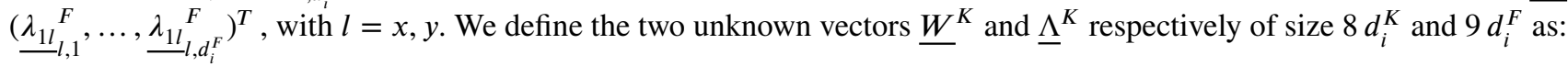

$$
\underline{W}^{K}=\left(\underline{\mathrm{u}}_{x}^{K}, \underline{\mathrm{u}}_{y}^{K}, \underline{\mathrm{w}}_{x}^{K}, \underline{\mathrm{w}}_{y}^{K}, \underline{\tau}_{x x}^{K}, \underline{\tau}_{y y}^{K}, \underline{\tau}_{x y}^{K}, \underline{\mathrm{p}}^{K}\right)^{T},
$$

and

$$
\underline{\Lambda}^{K}=\left(\underline{\lambda}_{1 x}^{\beta(K, 1)}, \underline{\lambda}_{1 x}^{\beta(K, 2)}, \underline{\lambda}_{1 x}^{\beta(K, 3)}, \underline{\lambda}_{1 y}^{\beta(K, 1)} \underline{\lambda}_{1 y}^{\beta(K, 2)}, \underline{\lambda}_{1 y}^{\beta(K, 3)}, \underline{\lambda}_{2}^{\beta(K, 1)}, \underline{\lambda}_{2}^{\beta(K, 2)}, \underline{\lambda}_{2}^{\beta(K, 3)}\right)^{T}
$$

where $\beta(K, f)$ is the global index of the $f$-th face of the element $K$. 
We also define the following elementary matrices $\mathbb{M}^{K}, \mathbb{D}_{v}^{K}, \mathbb{E}^{F}, \mathbb{J}_{v}^{F}$ of size $d_{i}^{K} \times d_{i}^{K}, \mathbb{F}^{F}, \mathbb{Q}_{v}^{F}$ of size $d_{i}^{K} \times d_{i}^{F}$, and $\mathbb{G}^{F}, \mathbb{U}_{v}^{F}$ of size $d_{i}^{F} \times d_{i}^{F}$ :

$$
\begin{aligned}
& \mathbb{M}_{i j}^{K}=\int_{K} \varphi_{i}^{K} \varphi_{j}^{K} d X, \quad \mathbb{D}_{v i j}^{K}=\int_{K} \varphi_{j}^{K} \frac{\partial \varphi_{i}^{K}}{\partial v} d X, \quad \mathbb{E}_{i j}^{F}=\int_{F} \varphi_{i}^{K} \varphi_{j}^{K} d S, \quad \mathbb{J}_{v i j}^{F}=\int_{F} \varphi_{i}^{K} \varphi_{j}^{K} n_{v} d S, \\
& \mathbb{F}_{i j}^{F}=\int_{F} \psi_{j}^{F} \varphi_{i}^{K} d S, \quad \quad \mathbb{Q}_{v i j}^{F}=\int_{F} \psi_{j}^{F} \varphi_{i}^{K} n_{v} d S, \quad \mathbb{G}_{i j}^{F}=\int_{F} \psi_{j}^{F} \psi_{j}^{F} d S, \quad \quad \mathbb{G}_{v i j}^{F}=\int_{F} \psi_{i}^{F} \psi_{j}^{F} n_{v} d S,
\end{aligned}
$$

with $v=x, y$. Moreover, we define four elementary source vectors of size $d_{i}^{K}$

$$
\left(\mathbb{C}_{1 x}^{K}\right)_{i}=\int_{K} f_{\mathrm{vol} x}^{K} \varphi_{i}^{K}, \quad\left(\mathbb{C}_{1 y}^{K}\right)_{i}=\int_{K} f_{\mathrm{vol} y}^{K} \varphi_{i}^{K}, \quad\left(\mathbb{C}_{2 x}^{K}\right)_{i}=\int_{K} g_{\mathrm{vol} x}^{K} \varphi_{i}^{K}, \quad\left(\mathbb{C}_{2 y}^{K}\right)_{i}=\int_{K} g_{\mathrm{vol} y}^{K} \varphi_{i}^{K} .
$$

Remark 2. In using straight-edge meshes, and Lagrangian polynomials for approximation spaces, the components of the above local matrices are computed by the standard techniques in which they are rewritten in terms of integrals on the reference element (triangle or the line interval $[0,1]$ ) with the reference Lagrangian polynomials.

\section{1 | Local problem}

Because of the size of the system of equations, we present the discretization of each equation of the local problem (16). Using the above representation of the local unknowns, each equation is written in terms of the matrices defined in (23), (for more details, see $\left.e^{(44}\right)$.

The equation of motion (16a) Taking $\bullet=x, y$, discretizing equation (16a) along the $x$ and $y$ component gives:

$$
\begin{aligned}
\int_{K} \mathrm{i} \omega \rho_{a}^{K} \mathrm{u}_{\bullet}^{K} \varphi_{i}^{K} & +\int_{K} \mathrm{i} \omega \rho_{f}^{K} \mathrm{w}_{\bullet}^{K} \varphi_{i}^{K}-\int_{K} \frac{\partial \tau_{x \bullet}^{K}}{\partial x} \varphi_{i}^{K}-\int_{K} \frac{\partial \tau_{\bullet y}^{K}}{\partial y} \varphi_{i}^{K}+\int_{\partial K} \gamma_{1} \mathrm{u}_{\bullet}^{K} \varphi_{i}^{K}-\int_{\partial K} \gamma_{1} \lambda_{1} \cdot \varphi_{i}^{K} \\
& +\int_{\partial K} \gamma_{3} \mathrm{p}_{h}^{K} n_{\bullet} \varphi_{i}^{K}-\int_{\partial K} \gamma_{3} \lambda_{2} n_{\bullet} \varphi_{i}^{K}=\int_{K} f_{\mathrm{vol}}^{K} \varphi_{i}^{K} .
\end{aligned}
$$

In terms of the local matrices defined in equation $(23)$ and the unknowns vectors in $(22)$, the above equations are

$$
\begin{aligned}
& \mathrm{i} \omega \rho_{a}^{K} \mathbb{M}^{K} \underline{\mathrm{u}}_{\bullet}^{K}+\mathrm{i} \omega \rho_{f}^{K} \mathbb{M}^{K} \underline{\mathrm{w}}^{K} \cdot-\left(\mathbb{D}_{x}^{K}\right)^{T} \underline{\tau}_{x \bullet}^{K}-\left(\mathbb{D}_{y}^{K}\right)^{T} \underline{\tau}_{\cdot}^{K},+\sum_{f=1}^{3} \gamma_{1} \mathbb{E}^{\beta(K, f)} \underline{\mathrm{u}}_{\bullet}^{K}-\sum_{f=1}^{3} \gamma_{1} \mathbb{F}^{\beta(K, f)} \underline{\lambda}_{-}^{\beta(K, f)} \\
& +\sum_{f=1}^{3} \gamma_{3} \rrbracket^{\beta(K, f)} \underline{\mathrm{p}}^{K}-\sum_{f=1}^{3} \gamma_{3} \mathbb{Q}_{\bullet}^{\beta(K, f)} \underline{\lambda}_{2}^{\beta(K, f)}=\mathbb{C}_{1 \bullet}^{K} .
\end{aligned}
$$

The second equation of motion (16b) Similarly, equation (16b) is discretized along $x$ and $y$ direction and gives in terms of the local matrices and the unknown vectors defined respectively in equations (23) and (22):

$$
\mathrm{i} \omega \rho_{f}^{K} \mathbb{M}^{K} \underline{\mathrm{u}}^{K}+\mathrm{i} \omega \rho_{\mathrm{dyn}}^{K} \mathbb{M}^{K} \underline{\underline{w}}^{K}-\mathbb{D}_{\cdot}^{K} \underline{\mathrm{p}}^{K}-\sum_{f=1}^{3} \mathbb{Q}_{\bullet}^{\beta(K, f)} \underline{\lambda}_{2}^{\beta(K, f)}=\mathbb{C}_{2 \bullet}^{K}, \quad \text { with } \bullet=x, y .
$$

The first constitutive law (16c) The constitutive law equation given in (16c) is discretized as well and the equations are expressed in terms of local matrices and the unknown vectors defined in equations (22) and (23):

$$
\begin{gathered}
\mathrm{i} \omega \mathbb{M}^{K} \underline{\tau}_{x x}^{K}+\mathrm{i} \omega \alpha_{11}^{K} \mathbb{M}^{K} \underline{\mathrm{p}}^{K}+C_{11}^{K} \mathbb{D}_{x}^{K} \underline{\mathrm{u}}_{x}^{K}+C_{13}^{K} \mathbb{D}_{y}^{K} \underline{\mathrm{u}}_{x}^{K}+C_{13}^{K} \mathbb{D}_{x}^{K} \underline{\mathrm{u}}_{y}^{K}+C_{12}^{K} \mathbb{D}_{y}^{K} \underline{\mathrm{u}}_{y}^{K} \\
-\sum_{f=1}^{3} \underline{\lambda}_{1 x}^{\beta(K, f)}\left(C_{11}^{K} \mathbb{Q}_{x}^{\beta(K, f)}+C_{13}^{K} \mathbb{Q}_{y}^{\beta(K, f)}\right)-\sum_{f=1}^{3} \underline{\lambda}_{1 y}^{\beta(K, f)}\left(C_{13}^{K} \mathbb{Q}_{x}^{\beta(K, f)}+C_{12}^{K} \mathbb{Q}_{y}^{\beta(K, f)}\right)=0,
\end{gathered}
$$




$$
\begin{gathered}
\mathrm{i} \omega \mathbb{M}^{K} \underline{\tau}_{y y}^{K}+\mathrm{i} \omega \alpha_{22}^{K} \mathbb{M}^{K} \underline{\mathrm{p}}^{K}+C_{12}^{K} \mathbb{D}_{x}^{K} \underline{\mathrm{u}}_{x}^{K}+C_{23}^{K} \mathbb{D}_{y}^{K} \underline{\mathrm{u}}_{x}^{K}+C_{23}^{K} \mathbb{D}_{x}^{K} \underline{\mathrm{u}}_{y}^{K}+C_{22}^{K} \mathbb{D}_{y}^{K} \underline{\mathrm{u}}_{y}^{K} \\
-\sum_{f=1}^{3} \underline{\lambda}_{1 x}^{\beta(K, f)}\left(C_{12}^{K} \mathbb{Q}_{x}^{\beta(K, f)}+C_{23} \mathbb{Q}_{y}^{\beta(K, f)}\right)-\sum_{f=1}^{3} \underline{\lambda}_{1 y}^{\beta(K, f)}\left(C_{23} \mathbb{Q}_{x}^{\beta(K, f)}+C_{22} \mathbb{Q}_{y}^{\beta(K, f)}\right)=0,
\end{gathered}
$$

and

$$
\begin{gathered}
\mathrm{i} \omega \mathbb{M}^{K} \underline{\tau}_{x y}^{K}+\mathrm{i} \omega \alpha_{12}^{K} \mathbb{M}^{K} \underline{\mathrm{p}}^{K}+C_{13}^{K} \mathbb{D}_{x}^{K} \underline{\mathrm{u}}_{x}^{K}+C_{33}^{K} \mathbb{D}_{y}^{K} \underline{\mathrm{u}}_{x}^{K}+C_{33}^{K} \mathbb{D}_{x}^{K} \underline{\mathrm{u}}_{y}^{K}+C_{23}^{K} \mathbb{D}_{y}^{K} \underline{\mathrm{u}}_{y}^{K} \\
-\sum_{f=1}^{3} \underline{\lambda}_{1 x}^{\beta(K, f)}\left(C_{13}^{K} \mathbb{Q}_{x}^{\beta(K, f)}+C_{33}^{K} \mathbb{Q}_{y}^{\beta(K, f)}\right)-\sum_{f=1}^{3} \underline{\lambda}_{1 y}^{\beta(K, f)}\left(C_{33}^{K} \mathbb{Q}_{x}^{\beta(K, f)}+C_{23}^{K} \mathbb{Q}_{y}^{\beta(K, f)}\right)=0 .
\end{gathered}
$$

The second constitutive law (16d) The discretization of equation (16d) gives in terms of the local matrices defined in equation (23) and the unknown vectors in (22):

$$
\begin{aligned}
\mathrm{i} \omega \mathbb{M}^{K} \underline{\mathrm{p}}^{K} & +M^{K}\left(\mathbb{D}_{x}^{K}\right)^{T} \underline{\mathrm{w}}_{x}^{K}+M^{K}\left(\mathbb{D}_{y}^{K}\right)^{T} \underline{\mathrm{w}}_{y}^{K}-\sum_{f=1}^{3} M^{K} \gamma_{2} \mathbb{E}^{\beta(K, f)} \underline{\mathrm{p}}^{K}+\sum_{f=1}^{3} M^{K} \gamma_{2} \underline{\lambda}_{2}^{\beta(K, f)} \mathbb{F}^{\beta(K, f)} \\
& -\sum_{f=1}^{3} M^{K} \gamma_{4}\left(\mathbb{J}_{x}^{\beta(K, f)} \underline{\mathrm{u}}_{x}^{K}+\mathbb{J}_{y}^{\beta(K, f)} \underline{\mathrm{u}}_{y}^{K}\right)+\sum_{f=1}^{3} M \gamma_{4}\left(\mathbb{Q}_{x}^{\beta(K, f)} \underline{\lambda}_{1 x}^{\beta(K, f)}+\mathbb{Q}_{y}^{\beta(K, f)} \underline{\lambda}_{1 y}^{\beta(K, f)}\right) \\
& -M^{K} \alpha_{11}^{K} \mathbb{D}_{x}^{K} \mathrm{u}_{x}^{K}-M^{K} \alpha_{12}^{K} \mathbb{D}_{y}^{K} \mathrm{u}_{y}^{K}+\sum_{f=1}^{3} M^{K}\left(\alpha_{11} \underline{\lambda}_{1 x}^{\beta(K, f)}+\alpha_{12}^{K} \underline{\lambda}_{1 y}^{\beta(K, f)}\right) \mathbb{Q}_{x}^{\beta(K, f)} \\
& -M^{K} \alpha_{12}^{K} \mathbb{D}_{y}^{K} \mathrm{u}_{x}^{K}-M^{K} \alpha_{22}^{K} \mathbb{D}_{x}^{K} \mathrm{u}_{y}^{K}+\sum_{f=1}^{3} M^{K}\left(\alpha_{12}^{K} \underline{\lambda}_{1 x}^{\beta(K, f)}+\alpha_{22}^{K} \underline{\lambda}_{1 y}^{\beta(K, f)}\right) \mathbb{Q}_{y}^{\beta(K, f)}=0 .
\end{aligned}
$$

Local linear system The local system obtained from the discretization of (16) can be written as:

$$
\mathbb{A}^{K} \underline{W}^{K}+\mathbb{B}^{K} \underline{\Lambda}^{K}=\mathbb{C}_{\text {source }}^{K},
$$

where $\underline{W}^{K}$ and $\underline{\Lambda}^{K}$ have been defined in $(22)$. In the above equation, $\mathbb{A}^{K}$ is of dimension $8 d_{i}^{K} \times 8 d_{i}^{K}$, $\mathbb{B}^{K}$ of size $8 d_{i}^{K} \times 9 d_{i}^{F}$, and $\mathbb{C}_{\text {source }}^{K}$ is the matrix of the external forces of dimension $8 d_{i}^{K}$.

$W^{K}$ is composed of 8 blocks corresponding to different types of unknowns, we hence decompose the elementary matrix $\mathbb{A}^{K}$ in 8 columns of size $8 d_{i}^{K} \times d_{i}^{K}$ :

$$
\mathbb{A}^{K}=\left(\begin{array}{llllllll}
\mathbb{A}_{1}^{K} & \mathbb{A}_{2}^{K} & \mathbb{A}_{3}^{K} & \mathbb{A}_{4}^{K} & \mathbb{A}_{5}^{K} & \mathbb{A}_{6}^{K} & \mathbb{A}_{7}^{K} & \mathbb{A}_{8}^{K}
\end{array}\right), \quad \text { that are detailed in appendix } \mathrm{A}
$$

Similarly, based on the structure of the unknown $\Lambda^{K}$ in 3 times 3 sub-blocks corresponding to the three Lagrange unknowns decomposed on the three faces of the triangle, we write $\mathbb{B}^{K}$ in 9 columns of size $8 d_{i}^{K} \times d_{i}^{F}$ :

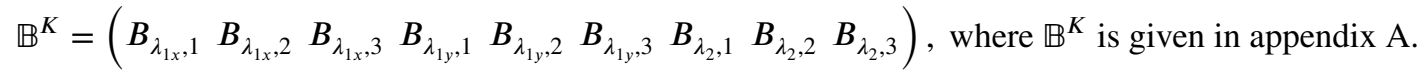

Finally the local source vector is:

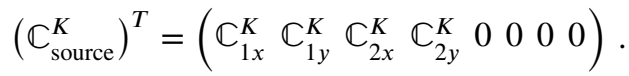

\section{2 | Transmission conditions}

Recall the transmission conditions given by equation (17):

$$
\sum_{F \in \mathcal{F}_{h}} \int_{F} \llbracket \hat{\boldsymbol{\tau}}_{h} n \rrbracket \cdot \eta=\sum_{F \in \mathcal{F}_{\mathrm{ext}}} \int_{F} f_{\mathrm{inc}} \cdot \eta,
$$

$$
\sum_{F \in \mathcal{F}_{h}} \int_{F} \llbracket \hat{\mathbf{w}}_{h} \cdot \boldsymbol{n} \rrbracket \xi=\sum_{F \in \mathcal{F}_{\text {ext }}} \int_{F} g_{\text {inc }} \xi,
$$


which are equivalent to

$$
\sum_{K \in \mathcal{T}_{h}} \int_{\partial K}\left(\hat{\boldsymbol{\tau}}_{h} \boldsymbol{n}\right) \cdot \eta=\sum_{F \in \mathcal{F}_{\text {ext }}} \int_{F} \boldsymbol{f}_{\text {inc }} \cdot \eta, \quad \text { (32a) } \quad \sum_{K \in \mathcal{T}_{h}} \int_{\partial K} \hat{\mathbf{w}}_{h} \cdot \boldsymbol{n} \xi=\sum_{F \in \mathcal{F}_{\text {ext }}} \int_{F} g_{\text {inc }} \xi .
$$

The above equations are expressed using the definition of the numerical traces on a face in equation (14) and the definition of the jump on an interior face in equation (9). Equation (32a) gives:

$$
\sum_{K \in \mathcal{T}_{h}} \int_{\partial K} \tau_{h}^{K} n^{K} \cdot \eta-\sum_{K \in \mathcal{T}_{h}} \int_{\partial K} S_{1}\left(\mathbf{u}_{h}^{K}-\lambda_{1}\right) \cdot \eta-\sum_{K \in \mathcal{T}_{h}} \int_{\partial K}\left(\mathrm{p}_{h}^{K}-\lambda_{2}\right) S_{3} n^{K} \cdot \eta=\sum_{F \in \mathcal{F}_{\text {ext }}} \int_{F} f_{\mathrm{inc}} \cdot \eta,
$$

and equation $32 \mathrm{~b}$ is expressed as:

$$
\sum_{K \in \mathcal{T}_{h}} \int_{\partial K} \mathbf{w}_{h}^{K} \cdot \boldsymbol{n}^{K} \xi-\sum_{K \in \mathcal{T}_{h}} \int_{\partial K}\left(\mathrm{p}_{h}^{K}-\lambda_{2}\right)\left(\boldsymbol{S}_{2} \boldsymbol{n}^{K}\right) \cdot \boldsymbol{n}^{K} \xi-\sum_{K \in \mathcal{T}_{h}} \int_{\partial K} \boldsymbol{S}_{4}\left(\mathbf{u}_{h}^{K}-\lambda_{1}\right) \cdot \boldsymbol{n}^{K} \cdot \xi=\sum_{F \in \mathcal{F}_{\text {ext }}} \int_{F} g_{\text {inc }} \xi .
$$

These equations are discretized on $(x, y)$ by decomposing the unknowns using equations $(20)$ and $(21)$ and replacing the test functions by the basis functions. They are then expressed in terms of the elementary matrices defined in 23 .

The first transmission equation 33a) The discretization of 33a along $x$ and $y$ direction gives, taking $\bullet=x, y$ is:

$$
\begin{aligned}
& \sum_{K \in \mathcal{T}_{h}} \int_{\partial K} \underline{\tau}_{-}^{K} n_{x}^{K} \varphi_{j}^{K} \psi_{i}^{\beta(K, f)} d S+\sum_{K \in \mathcal{T}_{h}} \int_{\partial K} \underline{\tau}_{\bullet}^{K} n_{y}^{K} \varphi_{j}^{K} \psi_{i}^{\beta(K, f)} d S-\sum_{K \in \mathcal{T}_{h}} \int_{\partial K} \gamma_{1} \varphi_{j}^{K} \underline{\mathrm{u}}_{\bullet}^{K} \psi_{i}^{\beta(K, f)} d S+\sum_{K \in \mathcal{T}_{h}} \int_{\partial K} \gamma_{1} \psi_{j}^{\beta(K, f)} \underline{\lambda}_{\bullet}^{K} \cdot \psi_{i}^{\beta(K, f)} d S \\
& -\sum_{K \in \mathcal{T}_{h}} \int_{\partial K} \gamma_{3} n_{\bullet}^{K} \underline{\mathrm{p}}^{K} \varphi_{j}^{K} \psi_{i}^{\beta(K, f)} d S+\sum_{K \in \mathcal{T}_{h}} \int_{\partial K} \gamma_{3} n_{\bullet}^{K} \underline{\lambda}_{2}^{K} \psi_{j}^{\beta(K, f)} \psi_{i}^{\beta(K, f)} d S=\sum_{F \in \mathcal{F}_{\text {ext }}} \int_{F} f_{\mathrm{inc}, \bullet} \psi_{i}^{\beta(K, f)} d S .
\end{aligned}
$$

This is expressed in terms of the local matrices defined in equation (23) and the unknowns vectors in 22) as:

$$
\begin{aligned}
\sum_{K \in \mathcal{T}_{h}}\left(\left(\mathbb{Q}_{x}^{\beta(K, f)}\right)^{T}\right. & \left.\underline{\tau}_{x_{\bullet}}^{K}+\left(\mathbb{Q}_{y}^{\beta(K, f)}\right)^{T} \underline{\tau}_{\bullet y}^{K}-\gamma_{1}\left(\mathbb{F}^{\beta(K, f)}\right)^{T} \underline{\mathrm{u}}_{\bullet}^{K}+\gamma_{1} \mathbb{G}^{\beta(K, f)} \underline{\lambda}_{\bullet \bullet}^{\beta(K, f)}-\gamma_{3}\left(\mathbb{Q}_{\bullet}^{\beta(K, f)}\right)^{T} \underline{\mathrm{p}}^{K}+\gamma_{3} \mathbb{H}_{\bullet}^{\beta(K, f)} \underline{\lambda}_{2}^{\beta(K, f)}\right) \\
& =\sum_{F \in \mathcal{F}_{\text {ext }}} \int_{F} f_{\mathrm{inc}, \bullet} \psi_{i}^{\beta(K, f)} d S
\end{aligned}
$$

The second transmission condition (33b) Similarly, the discretization of equation (33b) gives in terms of the local matrices and the unknown vectors defined respectively in equations 23] and 22]:

$$
\begin{aligned}
& \sum_{K \in \mathcal{T}_{h}}\left(\left(\mathbb{Q}_{x}^{\beta(K, f)}\right)^{T} \underline{\mathrm{w}}_{x}^{K}+\left(\mathbb{Q}_{y}^{\beta(K, f)}\right)^{T} \underline{\mathrm{w}}_{y}^{K}-\left(\mathbb{F}^{\beta(K, f)}\right)^{T} \underline{\mathrm{p}}^{K} \gamma_{2}-\left(\mathbb{F}^{\beta\left(K^{\prime}, g\right)}\right)^{T} \underline{\mathrm{p}}^{K^{\prime}} \gamma_{2}-\left(\mathbb{Q}_{x}^{\beta(K, f)}\right)^{T} \underline{\mathrm{u}}_{x}^{K} \gamma_{4}-\left(\mathbb{Q}_{y}^{\beta(K, f)}\right)^{T} \underline{\mathrm{u}}_{y}^{K} \gamma_{4}\right. \\
& \left.+\mathbb{E}_{x}^{\beta(K, f)} \underline{\lambda}_{1 x}^{K} \gamma_{4}+\mathbb{W}_{y}^{\beta(K, f)} \underline{\lambda}_{1 y}^{K} \gamma_{4}\right)=\sum_{F \in \mathcal{F}_{\text {ext }}} \int_{F} g_{\text {inc }} \psi_{i}^{\beta(K, f)} d S .
\end{aligned}
$$

System Let $N_{\text {face }}$ be the number of edges of the mesh. We define the global vector that gathers all the elementary Lagrange vectors:

$$
\underline{\Lambda}=\left(\underline{\lambda}_{1}^{1}, \underline{\lambda}_{2}^{1}, \ldots, \underline{\lambda}_{1}^{N_{\text {face }}}, \underline{\lambda}_{2}^{N_{\text {face }}}\right) .
$$

Define also the local trace operator $\mathcal{A}_{H D G}$ that links the local degrees of freedom on an element $K$ to the global degrees of freedom of the Lagrange multiplier $\underline{\Lambda}$. This means, for an element $K$, that

$$
\mathcal{A}_{H D G}^{K} \underline{\Lambda}=\underline{\Lambda}^{K} .
$$

The transmission conditions (35) and (36) are summed on all the faces of each element to give:

$$
\sum_{K \in \mathcal{T}_{h}}\left(\mathcal{A}_{H D G}^{K}\right)^{T}\left(\mathbb{P}^{K} \underline{W}^{K}+\mathbb{T}^{K} \mathcal{A}_{H D G}^{K} \underline{\Lambda}\right)=\mathbb{S}_{\mathrm{inc}},
$$

with $\mathbb{P}^{K}$ and $\mathbb{T}^{K}$ two matrices respectively of dimension $9 d_{i}^{F} \times 8 d_{i}^{K}$ and $9 d_{i}^{F} \times 9 d_{i}^{F}$ given in appendix A 


\section{3 | Solution methodology}

For an element $K$, we have built a local system:

$$
\mathbb{A} \underline{W}^{K}+\mathbb{B} \underline{\Lambda}^{K}=\mathbb{C}_{\text {source }}^{K}
$$

coupled with the transmission conditions

$$
\sum_{K \in \mathcal{T}_{h}}\left(\mathcal{A}_{H D G}^{K}\right)^{T}\left(\mathbb{P}^{K} \underline{W}^{K}+\mathbb{T}^{K} \mathcal{A}_{H D G}^{K} \underline{\Lambda}\right)=\mathbb{S}_{\mathrm{inc}} .
$$

Equation 38 is written as:

$$
\mathbb{A}^{K} \underline{W}^{K}=\mathbb{C}_{\text {source }}^{K}-\mathbb{B}^{K} \mathcal{A}_{H D G}^{K} \underline{\Lambda} .
$$

Assuming that the mesh is such that $\mathbb{A}^{K}$ can be inverted for each element, we have:

$$
\underline{W}^{K}=-\left(\mathbb{A}^{K}\right)^{-1} \mathbb{B}^{K} \mathcal{A}_{H D G}^{K} \underline{\Lambda}+\left(\mathbb{A}^{K}\right)^{-1} \mathbb{C}_{\text {source }}^{K} .
$$

Then $\underline{W}^{K}$ is replaced in (39) by its expression in (40):

$$
\sum_{K \in \mathcal{T}_{h}}\left(\mathcal{A}_{H D G}^{K}\right)^{T}\left(\mathbb{P}^{K}\left(\mathbb{A}^{K}\right)^{-1} \mathbb{C}_{\text {source }}^{K}-\mathbb{P}^{K}\left(\mathbb{A}^{K}\right)^{-1} \mathbb{B}^{K} \mathcal{A}_{H D G}^{K} \underline{\Lambda}+\mathbb{T}^{K} \mathcal{A}_{H D G}^{K} \underline{\Lambda}\right)=\mathbb{S}_{\text {inc }},
$$

which means,

$$
\sum_{K \in \mathcal{T}_{h}}\left(\mathcal{A}_{H D G}^{K}\right)^{T}\left(-\mathbb{P}^{K}\left(\mathbb{A}^{K}\right)^{-1} \mathbb{B}^{K}+\mathbb{T}^{K}\right) \mathcal{A}_{H D G}^{K} \underline{\Lambda}=-\sum_{K \in \mathcal{T}_{h}}\left(\mathcal{A}_{H D G}^{K}\right)^{T} \mathbb{P}^{K}\left(\mathbb{A}^{K}\right)^{-1} \mathbb{C}_{\text {source }}^{K}+\mathbb{S}_{\text {inc }} .
$$

The global problem writes:

$$
\mathbb{K} \underline{\Lambda}=\mathbb{S},
$$

with $\mathbb{K}=\sum_{K \in \mathcal{T}_{h}}\left(\mathcal{A}_{H D G}^{K}\right)^{T}\left(-\mathbb{P}^{K}\left(\mathbb{A}^{K}\right)^{-1} \mathbb{B}^{K}+\mathbb{T}^{K}\right)$ and $\mathbb{S}=-\sum_{K \in \mathcal{T}_{h}}\left(\mathcal{A}_{H D G}^{K}\right)^{T} \mathbb{P}^{K}\left(\mathbb{A}^{K}\right)^{-1} \mathbb{C}_{\text {source }}^{K}+\mathbb{S}_{\text {inc }}$.

The resolution can be divided in four steps, detailed in the following algorithm. First, we build the global matrix $\mathbb{K}$ and the source matrix $\mathbb{S}$. These calculations can be done element by element. Then the global system is resolved and the solution is constructed. We use the MUMPS direct solver for the resolution of the linear system, and this is the only step that is global.

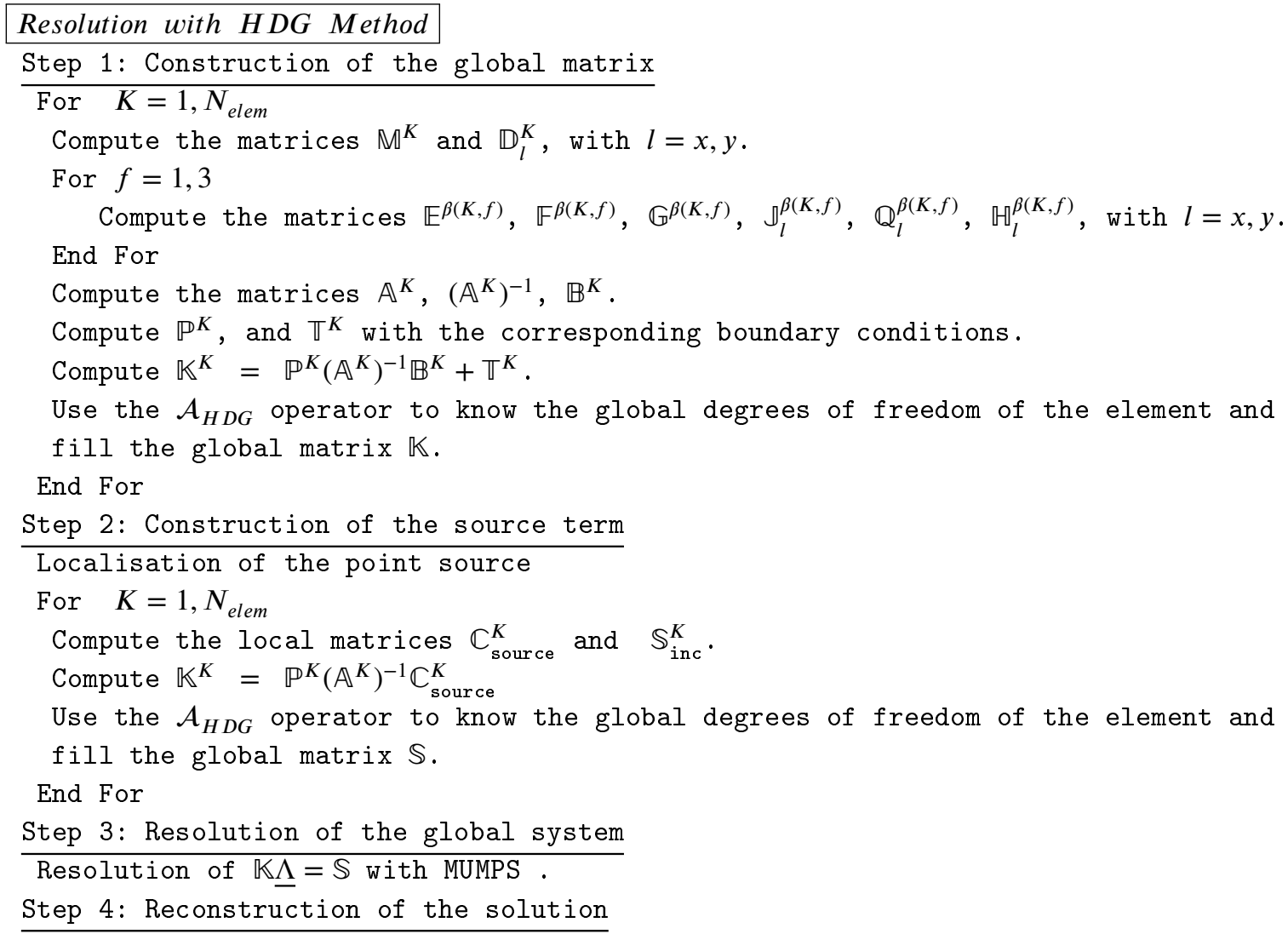




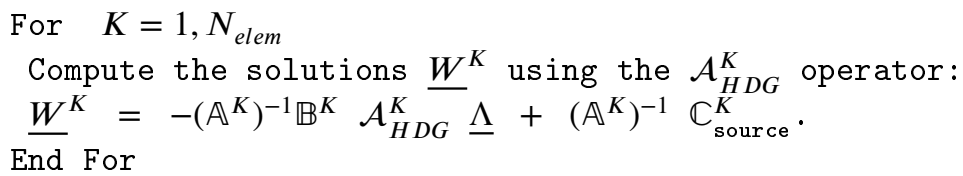

\section{5 | NUMERICAL RESULTS}

The goal of this section is to validate and analyze the performance of our numerical solver in Section 5.2 and to illustrate the behavior of the different types of poroelastic waves in Section 5.3 We will work with the geophysical media listed in Section 5.1 In Section 5.2 we first validate the code by comparing the HDG numerical solutions with reference ones given in ${ }^{38}$. We then carry out numerical tests to study the effect of stabilization parameters on the numerical error, the order of convergence, and the well-posedness of the problem. We also study the influence of the frequency on the well-posedness of the problem. Finally, in Section 5.3, we consider a domain composed of three horizontal layers and exhibit the three different types of waves in order to show that our methodology can reproduce the slow longitudinal-waves, even when their wavelength is much smaller than the two other ones and when the dissipation is high.

\section{1 | Parameterization of the computational domains and quantities of interest}

For numerical experiments, we work with sandstone, sand and shale. While sand is filled with a viscous fluid, shale and sandstone materials are filled with brine which is inviscid. We consider the case of isotropic and anisotropic sand whereas sandstone and shale are isotropic. We only specify when it is an anisotropic material. We list in Table 1 the physical parameters. Note that other parameters are obtained with those given, e.g. $\rho_{a}, \boldsymbol{\alpha}, M, H$, see Section 2

\begin{tabular}{|c|c|c|c|c|}
\hline Physical parameters & Sandstone & Shale & Isotropic Sand & Anisotropic Sand \\
\hline Porosity $\phi$ & 0.2 & 0.16 & 0.3 & 0.3 \\
\hline Fluid Density $\rho_{f}\left(10^{3} \mathrm{~kg} \cdot \mathrm{m}^{-3}\right)$ & 1.04 & 1.04 & 1 & 1 \\
\hline Solid Density $\rho_{s}\left(10^{3} \mathrm{~kg} \cdot \mathrm{m}^{-3}\right)$ & 2.5 & 2.21 & 2.6 & 2.6 \\
\hline Viscosity $\eta(m P a . s)$ & 0 & 0 & 1 & 1 \\
\hline Permeability $\kappa_{0}\left(\mu m^{2}\right)$ & 60 & 10 & 60 & 60 \\
\hline Tortuosity $\mathrm{t}$ & 2 & 2 & 3 & 2 \\
\hline Solid Bulk Modulus $\mathrm{k}_{\mathrm{s}}(G P a)$ & 40 & 7.6 & 35 & 35 \\
\hline Fluid Bulk Modulus $\mathrm{k}_{\mathrm{f}}(G P a)$ & 2.5 & 2.5 & 2.2 & 2.2 \\
\hline Frame Bulk Modulus $\mathrm{k}_{\mathrm{fr}}(G P a)$ & 20 & 6.6 & 0.4 & $C_{11}=1.067 \mathrm{GPa}$ \\
\hline Frame Shear Modulus $\mu_{\mathrm{fr}}(G P a)$ & 12 & 3.96 & 0.5 & $C_{22}=2.5 \mathrm{GPa}$ \\
\hline $\mathrm{v}_{\mathrm{P}}$ for $f=500 \mathrm{~Hz}\left(\mathrm{~m} \cdot \mathrm{s}^{-1}\right)$ & $4.2410^{3}$ & $2.4810^{3}$ & $\left(1.8810^{3}, 10.4\right)$ & $C_{33}=0.5 \mathrm{GPa}$ \\
\hline $\mathrm{v}_{\mathrm{S}}$ for $f=500 \mathrm{~Hz}\left(\mathrm{~m} \cdot \mathrm{s}^{-1}\right)$ & $2.3810^{3}$ & $1.4310^{3}$ & $\left(4.9310^{2}, 3.76\right)$ & $C_{12}=0.067 \mathrm{GPa}$ \\
\hline $\mathrm{v}_{\mathrm{B}}$ for $f=500 \mathrm{~Hz}\left(\mathrm{~m} \cdot \mathrm{s}^{-1}\right)$ & $1.0210^{3}$ & $1.1310^{3}$ & $\left(2.5710^{2}, 57.9\right)$ & $C_{13}=C_{23}=0 G P a$ \\
\hline
\end{tabular}

TABLE 1 Summary of the physical parameters that describe the different isotropic media. The parameters for sand are obtained from ${ }^{45}$ Table 1 , those for sandstone and shale from $\frac{13}{13}$ Table 5 . The values given here are the adimensional input values in the program.

Besides the geophysical parameters listed above, the admissible speed of propagation is also a key ingredient. In isotropic poroelastic media, there are three wavespeeds, the velocities of P (fast longitudinal), B (slow longitudinal) and S (shear) waves which are frequency dependent complex numbers. By $\underline{38}$ Proposition 3 , the admissible square slowness $s_{.}^{2}=s_{\cdot}^{2}(\omega)$ in an isotropic 
poroelastic medium are given by

$$
\begin{aligned}
\text { fast longitudinal wave } & 2 \mathrm{~s}_{\mathrm{P}}^{2}(\omega) & =\frac{\operatorname{tr} C(\omega)}{\operatorname{det} B}-\sqrt{\left(\frac{\operatorname{tr} C(\omega)}{\operatorname{det} B}\right)^{2}-4 \frac{\operatorname{det} A(\omega)}{\operatorname{det} B}}, \\
\text { slow longitudinal wave } & 2 \mathrm{~s}_{\mathrm{B}}^{2}(\omega) & =\frac{\operatorname{tr} C(\omega)}{\operatorname{det} B}+\sqrt{\left(\frac{\operatorname{tr} C(\omega)}{\operatorname{det} B}\right)^{2}-4 \frac{\operatorname{det} A(\omega)}{\operatorname{det} B}}, \\
\text { transverse wave } & \mathrm{s}_{\mathrm{S}}^{2}(\omega) & =\frac{\operatorname{det} A(\omega)}{\mu_{\mathrm{fr}} \rho_{\mathrm{dyn}}(\omega)} .
\end{aligned}
$$

In the above expressions,

$$
\begin{aligned}
\operatorname{tr} C(\omega) & =\rho_{\mathrm{dyn}}(\omega) H-2 \alpha M \rho_{f}+\rho_{a} M, \\
\operatorname{det} B & =M H-(\alpha M)^{2}=M\left(\lambda_{\mathrm{fr}}+2 \mu_{\mathrm{fr}}\right), \\
\operatorname{det} A(\omega) & =\rho_{a} \rho_{\mathrm{dyn}}(\omega)-\rho_{f}^{2},
\end{aligned}
$$

where we have introduced the additional parameter $H$ defined by $H=\frac{4}{3} \mu_{\mathrm{fr}}+\mathrm{k}_{\mathrm{s}}+\alpha^{2} M$. In expression (44), we have denoted by $\sqrt{\bullet}$ the square root branch that uses the principal value, i.e. for $z \in \mathbb{C} \backslash\{0\}$,

$$
\sqrt{z}:=\sqrt{|z|} e^{\mathrm{i} \frac{\operatorname{Arg}(z)}{2}}, \quad \operatorname{Arg}(z) \in(-\pi, \pi] .
$$

This means that $\operatorname{Re} \sqrt{ }>0$, while $\operatorname{Im} \sqrt{ }$ can be positive or negative. For the material listed in Table 1 , the various square slowness satisfy,

$$
\text { when } \eta>0, \operatorname{Res}^{2}>0,-\operatorname{Ims}_{\bullet}^{2}>0 ; \quad \text { when } \eta=0, \operatorname{Res}_{.}^{2}>0, \operatorname{Ims}_{\bullet}^{2}=0 .
$$

For exact numerical value, see 38 Table 2 .

The slowness s. is then defined as

$$
\text { s. }(\omega):=-\sqrt{s_{.}(\omega)^{2}} .
$$

For the material in consideration, this quantity satisfies

$$
\text { Res. }<0, \quad \text { Ims. } \geq 0 \text {. }
$$

From the slowness, we define the wave number $k_{\bullet}$, wave vector $\mathbf{k}_{\bullet}$ and complex-valued velocity $\mathrm{v}_{\bullet}$

$$
k_{\bullet}=\omega s_{\bullet}, \quad k_{\bullet}=k_{\bullet} \hat{\mathbf{d}}, \quad v_{\bullet}=\frac{1}{s_{.}}, \quad \bullet=P, B, S,
$$

With the above choices, and the property of $\mathrm{s}_{\mathbf{b}}$ in $(47)$, the quantity $e^{\mathrm{i} \omega t} e^{\mathbf{i k} \cdot \mathbf{x}}$ defines a travelling plane wave in direction $\widehat{\mathbf{d}}$ with phase speed $\frac{1}{|\operatorname{Res} .|}$ and is attenuated in the propagation direction, when $\eta>0$, at attenuation rate $\omega \mid \operatorname{Im~s.|}$.

We will work with the following plane wave solutions, $\mathrm{cf}^{-38}$,

$$
\mathcal{L}_{\text {poro }} \mathfrak{U}_{\mathrm{pw}}=0 \text { in } \mathbb{R}^{2}, \quad \text { with } \mathfrak{U}_{\mathrm{pw}}=\left(\mathbf{u}_{\mathrm{P}}, \mathbf{w}_{\mathrm{P}}, \boldsymbol{\tau}_{\mathrm{P}}, \mathrm{p}_{\mathrm{P}}\right)^{T},
$$

where

$$
\begin{aligned}
& \mathbf{u}_{\mathrm{P}}=e^{\mathrm{i} \mathbf{k}_{\mathrm{P}} \cdot \boldsymbol{x}}(\mathrm{i} \omega) \hat{\mathbf{d}}, \quad \mathbf{w}_{\mathrm{P}}=\beta_{\mathrm{P}} e^{\mathrm{i} \mathbf{k}_{\mathrm{P}} \cdot \boldsymbol{x}}(\mathrm{i} \omega) \hat{\mathbf{d}}, \\
& \tau_{\mathrm{P}}=\mathrm{i} \omega \mathrm{s}_{\mathrm{P}}(\omega) e^{i \mathbf{k}_{\mathrm{p}} \cdot \boldsymbol{x}}\left(2 \mu_{\mathrm{fr}} \hat{\mathbf{d}} \otimes \hat{\mathbf{d}}+\left(-\frac{2}{3} \mu_{\mathrm{fr}}+\mathrm{k}_{\mathrm{fr}}+M \alpha^{2}+\beta_{\mathrm{P}} \alpha M\right) \square\right), \\
& \mathrm{p}_{\mathrm{P}}=\mathrm{i} \omega \mathrm{s}_{\mathrm{P}}(\omega)\left(-M \beta_{\mathrm{P}}-M \alpha\right) e^{\mathrm{i} \mathbf{k}_{\mathrm{P}} \cdot \boldsymbol{x}} \text {. }
\end{aligned}
$$

In the above equations, $\mathbf{k}_{\mathrm{P}}=\omega \mathrm{s}_{\mathrm{P}}(\omega) \hat{\mathbf{d}}$ is the wave number, $\hat{\mathbf{d}}$ is the polarization with $|\hat{\mathbf{d}}|=1$. The slowness is given in equation 44a and the amplitude $\beta_{\mathrm{P}}$ of $\mathbf{w}_{\mathrm{P}}$ is

$$
\beta_{\mathrm{P}}(\omega)=-\frac{H \mathrm{~s}_{\mathrm{P}}^{2}(\omega)-\rho_{a}}{\alpha M \mathrm{~s}_{\mathrm{P}}^{2}(\omega)-\rho_{f}} .
$$

To assess the accuracy and the efficiency of the HDG numerical method, we will use the following quantities: 
- The relative numerical error $e_{h}(\mathfrak{U})$ is computed from the knowledge of the numerical solution denoted by $\mathfrak{U}_{\text {numeric }}$ and the reference solution $\mathfrak{U}_{\text {reference }}$, following the formula:

$$
e_{h}(\mathfrak{U})=\frac{\left\|\mathfrak{U}_{\text {numeric }}-\mathfrak{U}_{\text {reference }}\right\|_{2}}{\left\|\mathfrak{U}_{\text {reference }}\right\|_{2}}, \text { with } \quad\|\mathfrak{U}\|_{2}=\left(\sum_{K \in \mathcal{T}_{K}} \int_{K}|\mathfrak{U}|^{2}\right)^{\frac{1}{2}} .
$$

- To study the stability of discretization, we consider the condition number $\kappa$, defined, for a matrix $\mathbf{A}$ as $\kappa=\left\|\mathbf{A}^{-1}\right\|\|\mathbf{A}\|$, with $\|\mathbf{A}\|$ a matrix norm of $\mathbf{A}$. We employ MUMPS ${ }^{46}$ or LAPACK ${ }^{47}$ to compute $\kappa$ with the $L^{\infty}$ norm, $\|\mathbf{A}\|_{\infty}=$ $\max _{i}\left(\sum_{j=1}^{n}\left|a_{i j}\right|\right)$, where $a_{i j}$ is a coefficient of $\mathbf{A}$.

\section{2 | Validation of the numerical code and performance analysis}

Our numerical analysis in Section 5.2 is organized as follows.

1. In Section 5.2.1 to evaluate the accuracy of the HDG method, we consider a particular configuration on which the solution is known analytically, and use this to construct a reference solution to be compared with the numerical one.

2. We analyze in Section 5.2.2 the impact of the various numerical parameters on the order of convergence of the solution. We first show that the four penalization parameters are necessary in order to guarantee optimal convergence (at the order $p+1$ ) of all the unknowns. In applying this result, we then set the four penalization parameters to the same value denoted by $\gamma$, and show that the higher $\gamma$ is, the more accurate the solution is.

3. Next, we analyze the well-posedness of the local and global problem for a given choice of penalization parameters in Section 5.2.3 We show that a too high value of $\gamma$ deteriorates the condition number of the global and the local matrices. On the other hand, our numerical experiments show that the frequency does not influence the condition number, provided the mesh is chosen fine enough to guarantee a reasonable accuracy.

The characteristics of the computer used for the numerical experiments are the following: 2 Dodeca-core Haswell Intel $R$ Xeon $囚 \mathrm{E} 5-2680 \mathrm{v} 3$, at frequency $2.5 \mathrm{GHz}$, with 128 Go of memory (5.3 Go/core), a OmniPath $100 \mathrm{~Gb} / \mathrm{s}$ and a Infinipath $40 \mathrm{~Gb} / \mathrm{s}$.

\subsection{1 | Validation of the code}

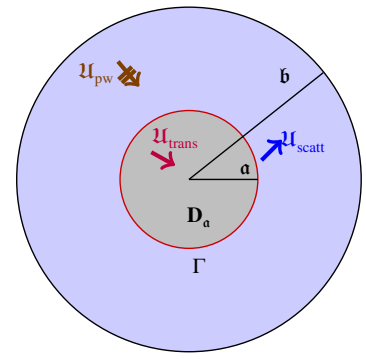

(a) Computational domain for problem in Equation 55

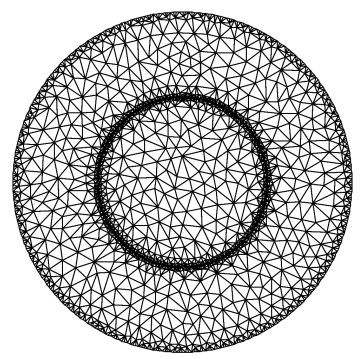

(b) Mesh of computational domain with straight-edge triangles

FIGURE 1 Set up for numerical solution $\mathfrak{U}_{\text {reference }}^{N}$ which approximates $\mathfrak{U}_{\text {analytic }}$ to machine precision on disc-shaped computation domain $\mathcal{D}_{\mathfrak{b}}$. The latter is the total wave in the scattering problem of a planewave $\mathfrak{U}_{\mathrm{pw}}$ by a penetrable disc-shaped obstacle represented by $\mathcal{D}_{\mathfrak{a}}$, given by equation $(52)$ (with the penetrable condition given by eq. (52b) on $r=\mathfrak{a}$ ).

For the validation of the code, we use a reference solution that we construct as an accurate approximation of an analytic solution. To have an analytic solution, we have to restrict ourselves to a particular configuration. We actually consider the case 
of a porous disc-shaped solid inclusion of radius $r=\mathfrak{a}$, denoted by $\mathcal{D}_{\mathfrak{a}}$, embedded in an infinite medium $\left(\mathbb{R}^{2} \backslash \mathcal{D}_{\mathfrak{a}}\right)$ made up of a different poroelastic material. We assume that there is a plane wave $\mathfrak{U}_{\mathrm{pw}}$ as defined in 49 ) which propagates in the exterior domain and comes across the obstacle $\mathcal{D}_{\mathfrak{a}}$. Then $\mathfrak{U}_{\text {analytic }}$ is the wavefield that arises from scattering of the incident plane wave. It satisfies the following problem:

$$
\begin{gathered}
\mathcal{L}_{\text {poro }} \mathcal{U}_{\text {analytic }}=0 \quad \text { in } \mathbb{R}^{2} \quad ; \quad \mathcal{U}_{\text {analytic }}-\mathcal{U}_{\mathrm{pw}} \text { is outgoing, } \\
\mathbf{u}^{(I)}-\mathbf{u}^{(I I)}=0, \quad \mathrm{p}^{(I)}-\mathrm{p}^{(I I)}=0, \quad\left(\mathbf{w}^{(I)}-\mathbf{w}^{(I I)}\right) \cdot \boldsymbol{n}=0, \quad\left(\boldsymbol{\tau}^{(I)}-\boldsymbol{\tau}^{(I I)}\right) \boldsymbol{n}=0 \quad, \quad \text { on } \partial \mathcal{D}_{\mathfrak{a}} .
\end{gathered}
$$

By 'outgoing', we mean in the sense of Definition 2 in ${ }^{38}$. In the transmission condition $52 \mathrm{~b}$ ), the unit normal vector is denoted by $\boldsymbol{n}$, and it is outwardly directed to the exterior of the inclusion $\mathcal{D}_{\mathbf{a}}$. Each condition is expressed in terms of the components of the restriction of the analytic solution, $\mathfrak{U}^{(I)}=\left.\mathfrak{U}_{\text {analytic }}\right|_{\mathcal{D}_{\mathfrak{a}}}$, and $\mathfrak{U}^{(I I)}=\left.\mathfrak{U}_{\text {analytic }}\right|_{\mathbb{R}^{2} \backslash \mathcal{D}_{\mathfrak{a}}}$ to the obstacle and the exterior domain respectively. The solution to (52) is then given by

$$
\mathfrak{U}_{\text {analytic }}=\mathfrak{U}_{\mathrm{pw}}+\mathfrak{U}_{\text {scatt }}, \text { in } \mathbb{R}^{2} \backslash \mathcal{D}_{\mathfrak{a}}, \quad \mathfrak{U}_{\text {analytic }}=\mathfrak{U}_{\text {trans }}, \text { in } \mathcal{D}_{\mathfrak{a}},
$$

with the scattered field $\mathfrak{U}_{\text {scatt }}$ and transmitted field $\mathfrak{U}_{\text {trans }}$ described explicitly in form of harmonic expansions, cf. Eq. (9.3) and (9.5) of $\frac{38}{38}$, see also $\frac{48}{4}$ Appendix A. These are infinite series whose coefficients are expressed in terms of the Hankel function of the first kind or Bessel J functions. In practice, we only keep the first $N$ terms of the infinite series, with $N \geq 2|\mathbf{k}| \mathfrak{a}+1^{49}$. By this way, we obtain a reference solution denoted by $\mathfrak{U}_{\text {reference }}^{N}$ and defined by:

$$
\mathfrak{U}_{\text {reference }}^{N}=\mathfrak{U}_{\mathrm{pw}}+\mathfrak{U}_{\text {scatt }}^{N}, \text { in } \mathbb{R}^{2} \backslash \mathcal{D}_{\mathfrak{a}}, \quad \mathfrak{U}_{\text {reference }}^{N}=\mathfrak{U}_{\text {trans }}^{N}, \text { in } \mathcal{D}_{\mathfrak{a}},
$$

and the exponent indicates that we keep the $N$ first terms in each of the series of the analytic scattered and transmitted fields. In our experiments, $N=50$, which verifies the above condition, and hence gives a very good approximation of the infinite series $2^{2}$ For the HDG-reference comparison, we will work with a bounded computational domain, see Figure 1(a) We assume that the obstacle is made up of sand, while the surrounding material is sandstone, cf. Table 1 for the values of their physical parameters. Here, we will work with boundary value of type 1 defined on a concentric disc of radius $\mathfrak{b}$, denoted by $\mathcal{D}_{\mathfrak{b}}$, which contains the aforementioned sand obstacle, and use the restriction of $\mathfrak{U}_{\text {reference }}^{N}$ to $\mathcal{D}_{\mathfrak{b}}$ as a reference solution. We introduce the following boundary value problem of type 1

$$
\mathcal{L}_{\text {poro }} \mathfrak{U}=0 \quad \text { in } \mathcal{D}_{\mathfrak{b}} \quad, \quad \mathcal{T}_{1} \mathfrak{U}=\mathcal{T}_{1} \mathfrak{U}_{\text {reference }}^{N} \text { on } r=\mathfrak{b},
$$

set on a disc of radius $\mathrm{b}$, denoted by $\mathcal{D}_{b}$, which is concentric with and contains the obstacle $\mathcal{D}_{\mathfrak{a}}$. Then the reference solution $\mathfrak{U}_{\text {reference }}^{N}$ is the unique solution to [55] except at eigenvalue frequencies. To construct the numerical solution, we will apply HDG method to 55 and compare the obtained numerical solution $\mathfrak{U}_{\text {hdg }}$ to $\left.\mathfrak{U}_{\text {reference }}^{N}\right|_{\mathcal{D}_{\mathfrak{6}}}$. In this approach, our HDG unknown in the exterior region is the 'total' wavefield (thus is compared with $\mathfrak{U}_{\mathrm{pw}}+\mathfrak{U}_{\mathrm{scatt}}^{N}$ ), and in the interior, it is the 'transmitted' one (thus compared with $\mathfrak{U}_{\text {trans }}^{N}$ ). Note that for computing the HDG solution, we use a mesh that is refined near the external boundary and the internal interface to minimize the geometrical errors without using curved elements (see Fig. 11(b). For the case of a straight boundary, we have observed that we do not need to refine the mesh.

Figure 2 shows the reference and numerical solutions for the test-case using the mesh displayed in Figure 1(b) and order 3 of discretization. The relative errors between reference and numerical solutions are given in Table 2 The stabilization parameters $\left(\gamma_{1}, \gamma_{2}, \gamma_{3}, \gamma_{4}\right)$, cf. (18) are equal to 1 . We can see that for each component, the error is small.

\begin{tabular}{|c|c|c|c|c|c|c|c|}
\hline$e_{h}\left(\mathrm{u}_{x}\right)$ & $e_{h}\left(\mathrm{u}_{y}\right)$ & $e_{h}\left(\mathrm{w}_{x}\right)$ & $e_{h}\left(\mathrm{w}_{y}\right)$ & $e_{h}\left(\tau_{x x}\right)$ & $e_{h}\left(\tau_{y y}\right)$ & $e_{h}\left(\tau_{x y}\right)$ & $e_{h}(\mathrm{p})$ \\
\hline 0.53 & 0.70 & 0.53 & 0.70 & 0.41 & 0.44 & 1.2 & 1.1 \\
\hline
\end{tabular}

TABLE 2 Relative errors (\%) on the components of the unknowns of the HDG method for the experiment shown in Figure 2 For an incident $\mathrm{P}$ wave, at frequency $f=500 \mathrm{~Hz}$ with $\theta=10^{\circ}$ and boundary conditions of type 1 . 


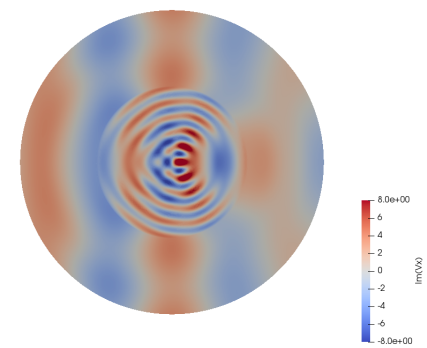

(a) Numerical solution

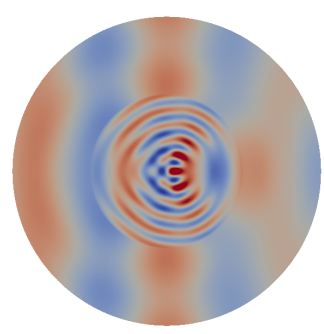

(b) Reference solution

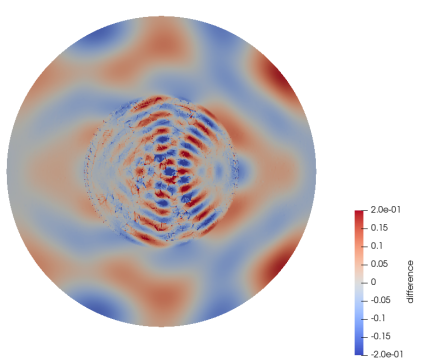

(c) Difference

FIGURE 2 Numerical and reference solutions for the scattering of a plane wave by a penetrable poroelastic inclusion at frequency $f=500 \mathrm{~Hz}$. The result is the imaginary part of the horizontal solid velocity. The resolution of the global system takes 6.52 seconds (CPU time), and it needs $677 \mathrm{MB}$ of memory to solve the global system.

\subsubsection{Impact of the stabilization parameters on the accuracy of the numerical solution}

In the previous section, we have carried out numerical experiments with all the stabilization parameters $\left(\gamma_{1}, \gamma_{2}, \gamma_{3}, \gamma_{4}\right)$, cf. (18), equal to 1 . In this section, we want to see the possible effects of various combinations of $\gamma_{i}$ on the order of convergence of the method, and then on the numerical error. For that purpose, we consider a homogeneous disc-shaped domain of radius $r=\mathfrak{b}$, where $\mathfrak{b}=10 \mathrm{~m}$ in the numerical tests, using boundary conditions of type 1 ,

$$
\mathcal{L}_{\text {poro }} \mathfrak{U}=0 \text { in } \mathcal{D}_{\mathfrak{b}}, \quad \mathcal{T}_{1} \mathfrak{U}=\mathcal{T}_{1} \mathfrak{U}_{\mathrm{pw}} \text { on } r=\mathfrak{b} .
$$

In this way, the reference (analytic) solution is given by $\mathfrak{U}_{\mathrm{pw}}$ defined in 50 . This study will show that if all the parameters are non zero, the relative error is always below $0.15 \%$ for $p=3$.

Order of convergence First, we want to display the order of convergence of the method in terms of the size of mesh. For that, we use four meshes with straight edges generated by the software Triangle. The refinement of a given mesh consists in dividing each of its cells into four cells. The characteristics of the meshes are summarized in Table 3 For the analysis, we introduce the definition of the size of the mesh $h$ which corresponds to the longest edge of the elements in the mesh. In Table 4, we show the

\begin{tabular}{|l|c|c|c|c|}
\hline & Mesh 1 & Mesh 2 & Mesh 3 & Mesh 4 \\
\hline Size of the mesh $h(\mathrm{~m})$ & 1.514 & 0.757 & 0.379 & 0.189 \\
Number of elements & 3270 & 13080 & 52320 & 209280 \\
\hline
\end{tabular}

TABLE 3 Characteristics of the meshes involved in the convergence analysis. The size of the mesh is the longest edge of the elements in the mesh.

results of the order of convergence for all four unknowns with varying degrees of stabilization. We illustrate the convergence curves for the case with full and then zero stabilization, in Figure 3(a) and 3(b) In these figures, we depict the relative error and thus the convergence order for the $x$-component $\mathrm{u}_{x}$ as a function of the size of the mesh. We have the following observations.

- For the case of full stabilization with all $\gamma_{i}=1$, we observe that the convergence order for $\mathrm{u}_{x}$ is equal to $p+1$, see also Remark 3 We also note that the same order of convergence is obtained for the other components of the numerical solution. The same result is obtained with other (equally set) values of the parameters, for instance when they are all equal to 0.1 . This result corroborates the ones obtained in ${ }^{37}$ with a different HDG formulation.

- In the case of only partial stabilization (i.e. some or all of the $\gamma_{i}$ are set to zero), one obtains a reduced order of convergence. It can occur that some components of the numerical solution keep an optimal order of convergence, while it is decreased for others, cf. Table 4 The extreme case is when all $\gamma_{i}=0$, the loss of convergence is observed for all unknown variables, cf. Figure 3(b) 


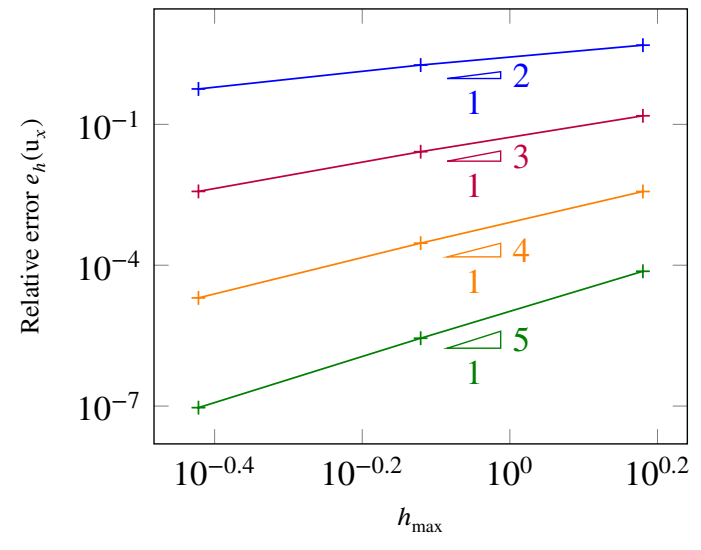

(a) Stabilization parameters set to 1

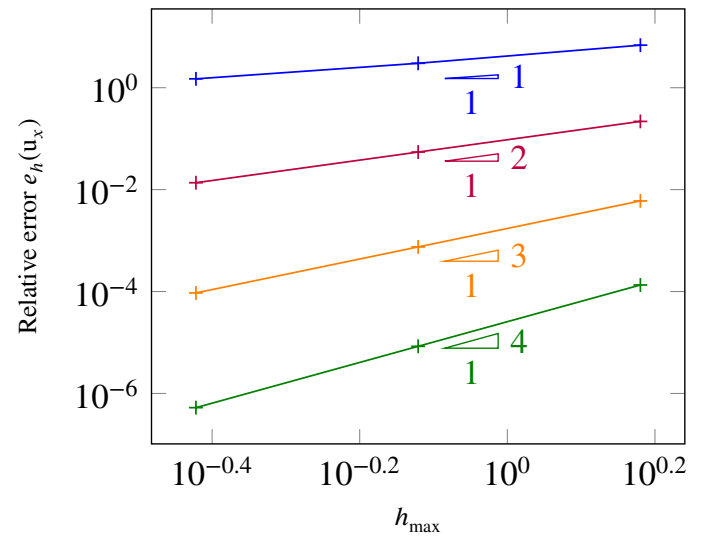

(b) Stabilization parameters set to 0

FIGURE 3 Convergence curves of HDG method (component $\mathrm{u}_{x}$ ) for several orders of interpolation. Results for order 1 are plotted in blue $\square$ for order 2 in purple $\square$, for order 3 in orange $\square$ and for order 4 in green $\square$ Figure $3(\mathrm{a})$ shows the results with the four stabilization parameters set to 1 and figure $3(\mathrm{~b})$ with the four stabilization parameters set to 0 . As expected, when we use the four stabilization parameters, we obtain convergence of order $p+1$, while we lose one order of convergence if we do not have the stabilization parameters, as for classical DG method without stabilization parameters.

- On the other hand, for some combinations of values of the stabilization parameters (not shown in Table 4 e.g. when $\left(\gamma_{1}, \gamma_{2}\right.$, $\left.\left.\gamma_{3}, \gamma_{4}\right)=(1,1,0,0)\right)$, the numerical solution is not accurate with numerical error attaining $200 \%$ and not decreasing even with higher degree of approximation and/or mesh refinement. However, we have observed that if the four stabilization parameters are different from zero, the numerical method is accurate with an optimal convergence order.

Remark 3. In Cockburn et al ${ }^{24}$, it is shown that in the case of linear elasticity, the order of convergence of the displacement can be upgraded to $p+2$ thanks to an element-per-element post-processing technique. We did not try to apply this idea to the Biot's system. An alternative to the post-processing is to increase the polynomial degree of two unknowns and to use a projection in the variational formulation, see 37 .

\begin{tabular}{|c|c|c|c|c|c|c|c|c|}
\hline $\begin{array}{c}\text { Stabilization parameters } \\
\left(\gamma_{1}, \gamma_{2}, \gamma_{3}, \gamma_{4}\right)\end{array}$ & 1111 & 1110 & 1011 & 1010 & 0000 & 0101 & 0100 & 0001 \\
\hline $\mathbf{u}$ & $p+1$ & $p+1$ & $p+1$ & $p+1$ & $p$ & $p$ & $p$ & $p$ \\
\hline $\mathbf{w}$ & $p+1$ & $p$ & $p+1$ & $p+1$ & $p$ & $p+1$ & $p$ & $p$ \\
\hline$\tau$ & $p+1$ & $p+1$ & $p$ & $p+1$ & $p$ & $p+1$ & $p+1$ & $p+1$ \\
\hline $\mathrm{p}$ & $p+1$ & $p+1$ & $p$ & $p$ & $p$ & $p+1$ & $p+1$ & $p$ \\
\hline
\end{tabular}

TABLE 4 Summary of the convergence order of the HDG method depending on the value of the stabilization parameters, defined in (18). The four parameters are necessary to obtain the optimal order of convergence on all the unknowns.

Numerical errors For the rest of the numerical tests, we will set all $\gamma_{i}=\gamma$. We now study the influence of this value $\gamma$ on the numerical error. Figure 4 shows the relative error as a function of the stabilization parameters, in the case of a polynomial approximation of order 3. The solid velocity $\mathbf{u}$ behaves in the same way as the elastic solid velocity observed in 29 Fig 3.4.7 p.89. We also note that the relative fluid velocity $\mathbf{w}$ and the pressure $\mathrm{p}$ seem to behave in a similar way, however of a different pattern from $\mathbf{u}$, and which is also different from that of $\boldsymbol{\tau}$. To arrive at a conclusion from these different patterns, we consider the mean value of the relative errors, depicted in Fig. 5 and defined as,

$$
e_{h}^{\text {mean }}=\frac{\sum_{u \in S}\left\|u_{\text {numeric }}-u_{\text {reference }}\right\|_{2}}{\sum_{u \in S}\left\|u_{\text {reference }}\right\|_{2}} \quad, \quad S=\left\{\mathrm{u}_{x}, \mathrm{u}_{y}, \mathrm{w}_{x}, \mathrm{w}_{y}, \tau_{x x}, \tau_{y y}, \tau_{x y}, \mathrm{p}\right\} .
$$




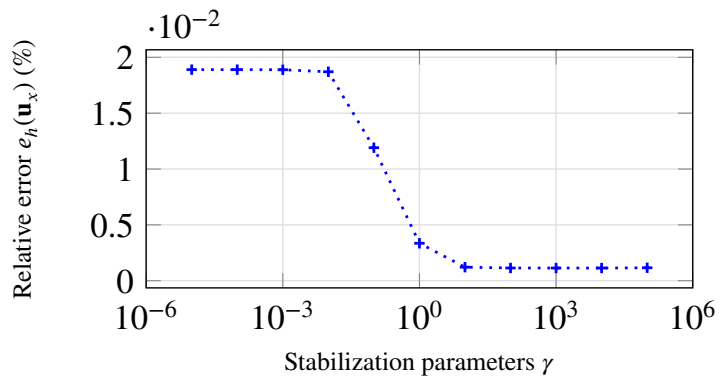

(a) $\mathrm{u}_{x}$

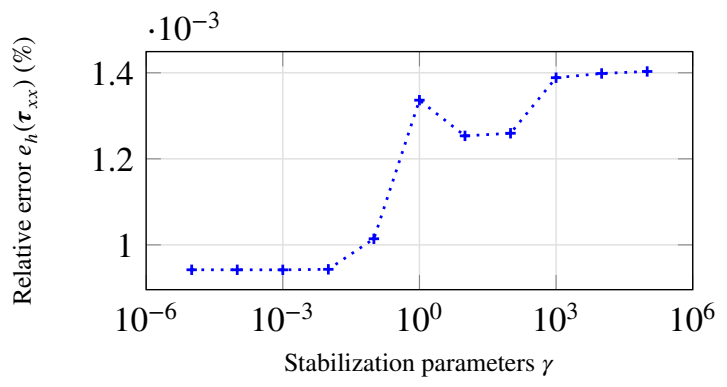

(c) $\tau_{x x}$

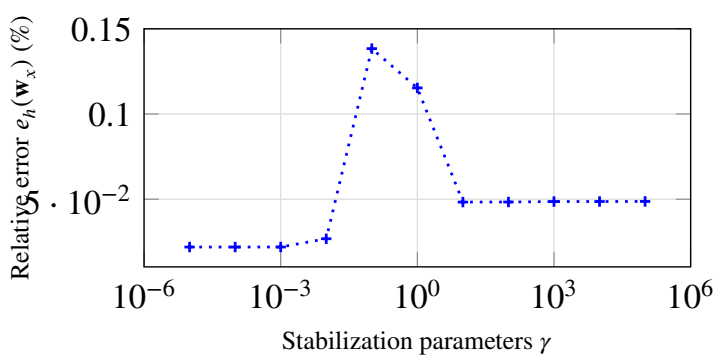

(b) $\mathrm{w}_{x}$

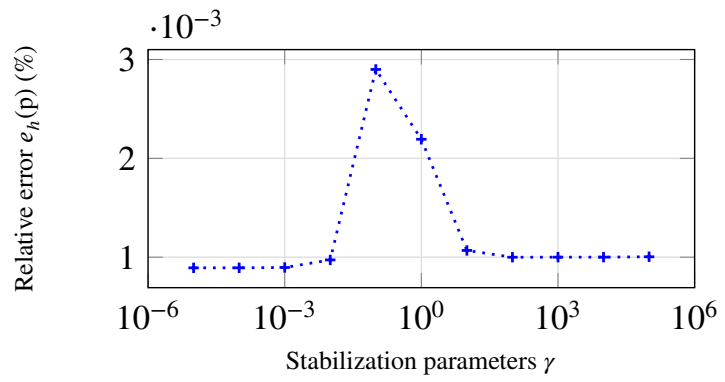

(d) $\mathrm{p}$

FIGURE 4 Relative error in L2-norm of HDG method (\%) depending on the stabilization parameters compared with reference solution developed in ${ }^{38}$. The stabilization parameters act differently on each unknown. When it is high, it improves the accuracy on $\mathbf{u}$, while when it is low, it improves the accuracy on $\tau$. It is important to note that the impact is never dramatic : the ratio between the maximal and the minimal error is less than 20 .

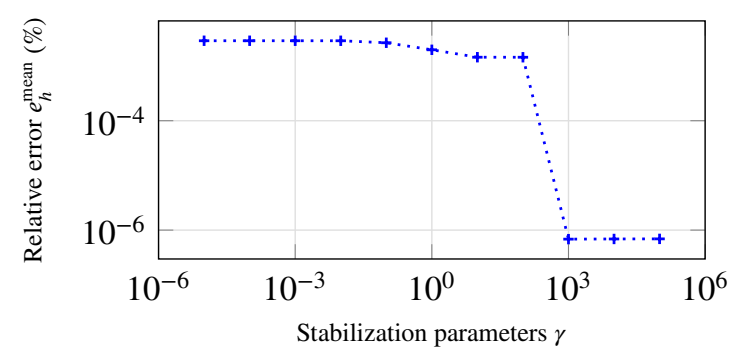

FIGURE 5 Mean relative error (\%) of the HDG method, depending on the stabilization parameters in semi-log scale. We defined this mean error (equation (57)) in order to identify more precisely the impact of the penalization parameters. This curve indicates that this parameter should be the highest possible

We observe that the mean error is less than $0.1 \%$ when the stabilization parameter is equal to at least $10^{3}$. We also note that the same high level of accuracy is guaranteed when the four stabilization parameters are less than $10^{-3}$.

\subsection{3 | Analysis of the well-posedness}

In this section, also working with problem (56), we continue the numerical study of well-posedness by analyzing the influence of the value of stabilization parameter $\gamma$ and the frequency on the condition number of the linear system. The latter is an indication of the well-posedness of the problem.

Influence of the stabilization parameters We first consider this question on the local level for the local matrix $\mathbb{A}^{K}$ and then for the global matrix $\mathbb{K}$. 


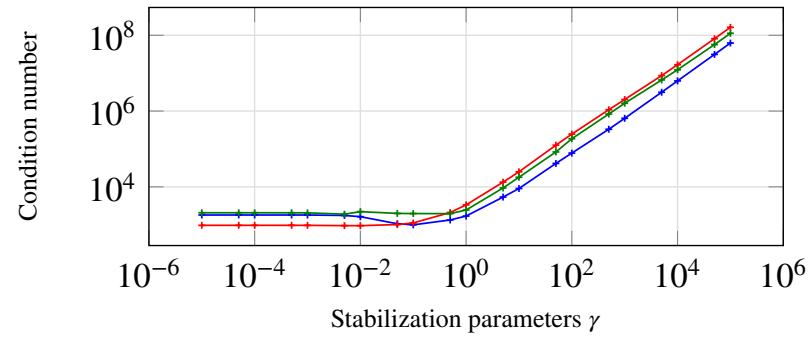

(a) P1

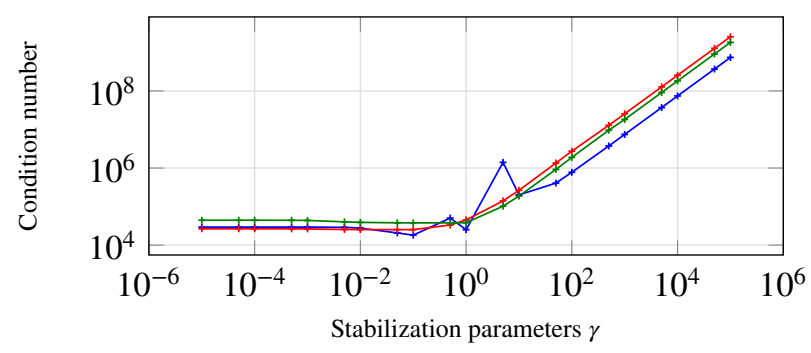

(c) P3

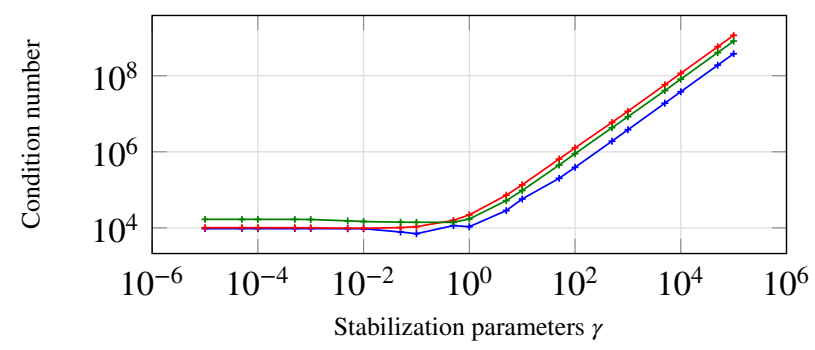

(b) P2

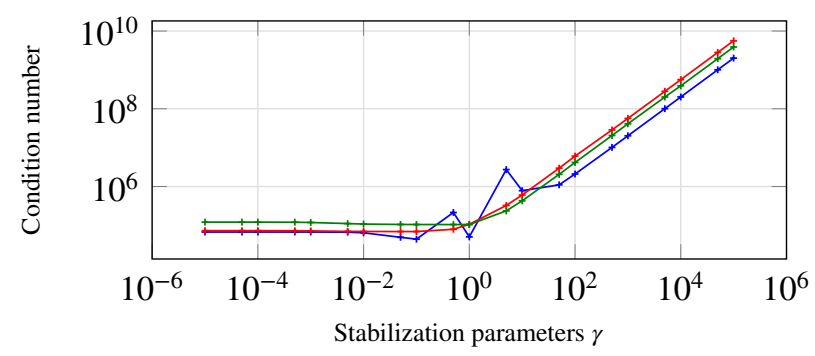

(d) P4

FIGURE 6 Maximal condition number (infinity norm) on all the elementary matrices $\mathbb{A}^{K}$ as a function of the stabilization parameters, for three materials and four interpolation orders at frequency $f=500 \mathrm{~Hz}$. The four stabilization parameters are set at the same value. The characteristics of the media are presented in table 1 The results for sand are represented in blue $\square$ for shale in red $\square$ and for sandstone in green $\square$. When the stabilization parameter is above 1, the condition number starts to increase. This shows that we should not set it too high.

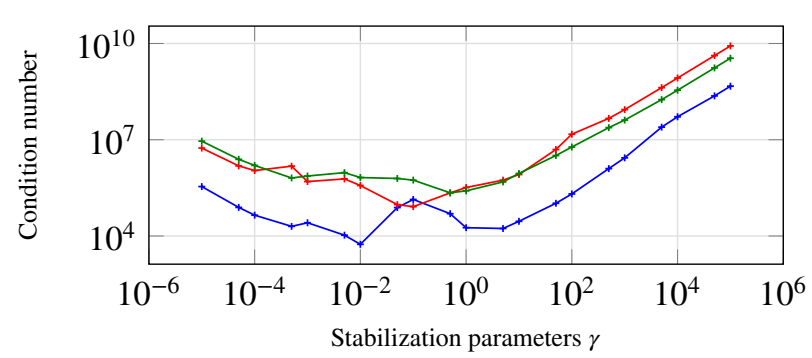

(a) P1

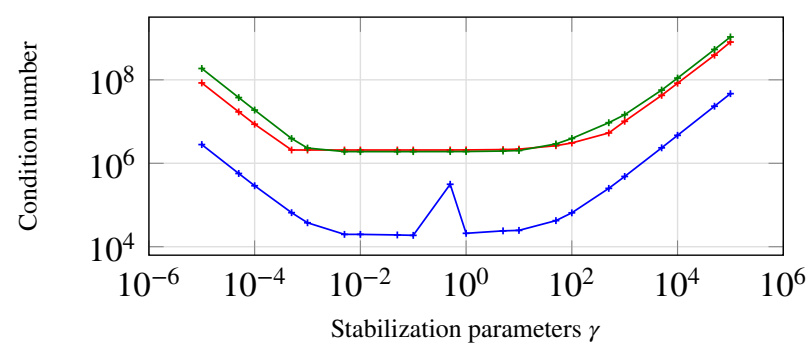

(c) P3

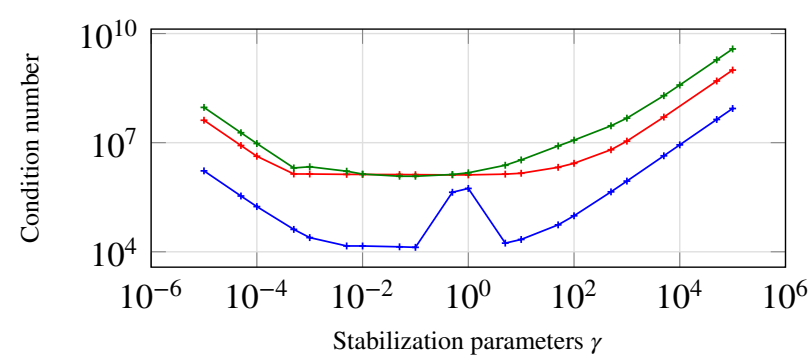

(b) P2

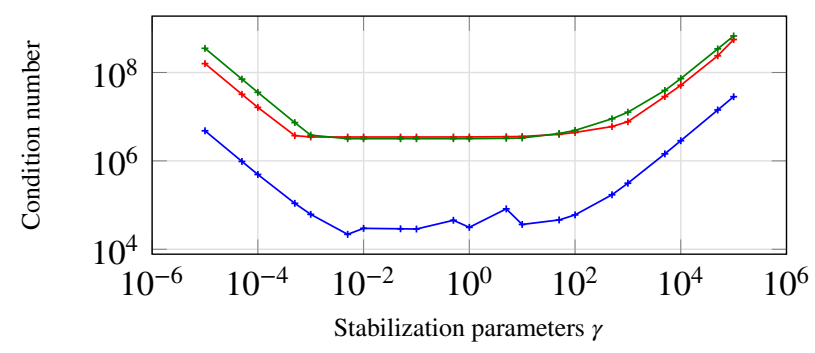

(d) P4

FIGURE 7 Condition number (infinity-norm) of the global matrix $\mathbb{K}$ as a function of the stabilization parameters, for three materials and four interpolation orders at frequency $f=500 \mathrm{~Hz}$. The four stabilization parameters are set at the same value. The characteristics of the media are presented in table 1 The results for sand are represented in blue $\square$, for shale in red $\square$ and for sandstone in green $\square$ 
- For the reconstruction of $\underline{W}^{K}$, we have to invert the elementary matrix $\mathbb{A}^{K}$, see equation 407 . Hence we study the condition number of $\mathbb{A}^{K}$ to evaluate if the calculation of $\left(\mathbb{A}^{K}\right)^{-1}$ is problematic. If the condition number is too high, the inversion will be less accurate. Since this inversion is performed on every element of the mesh, we consider the maximum value of the condition number, $C_{T_{h}}=\max _{K \in T_{h}}\left\|\left(A^{K}\right)^{-1}\right\|\left\|A_{K}\right\|$. For three different media and four different interpolation orders, we show the maximal condition number of $\mathbb{A}^{K}$ for every element of the mesh in infinity norm as a function of the values of the stabilization parameters in Figure 6 We observe that for all interpolation orders, the condition number increases with the stabilization parameters. We also note that the variations are similar for the different interpolation orders, also the condition number increases with the interpolation order, however staying bounded below $10^{10}$.

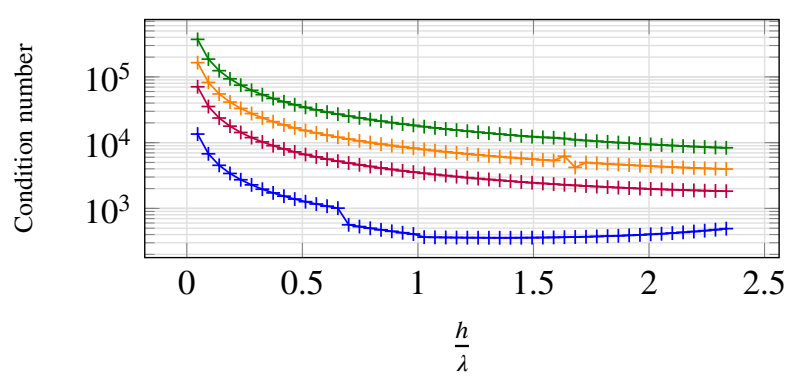

FIGURE 8 Condition number (infinity-norm) of the matrix $\mathbb{A}^{K}$ for one element function of $\frac{h}{\lambda_{\mathrm{B}}}$, for shale and four interpolation orders. The characteristics of the medium are presented in table 1 . The four stabilization parameters are set to 1 . Results for order 1 are plotted in blue $\square$ for order 2 in purple $\square$ for order 3 in orange $\square$ and for order 4 in green $\square$ The condition number is always below $10^{8}$, so that the matrix is easily invertible with a direct solver such as Lapack.

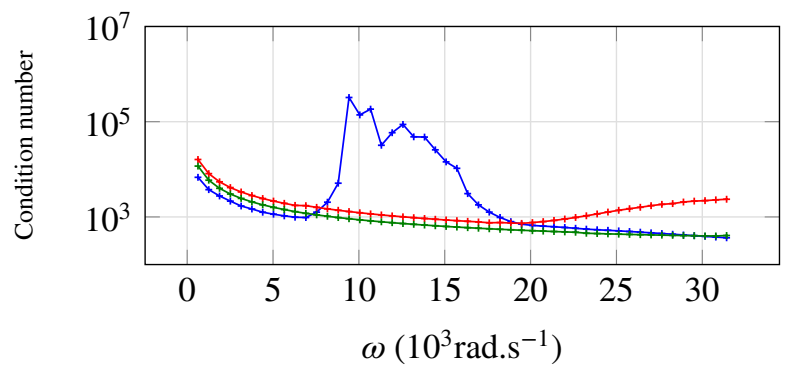

(a) P1

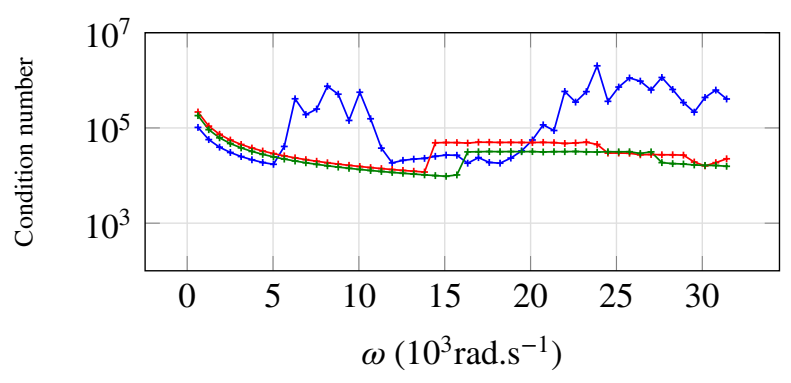

(c) P3

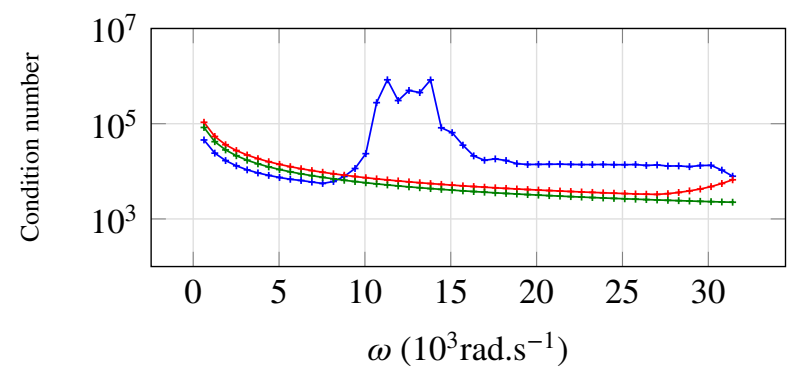

(b) P2

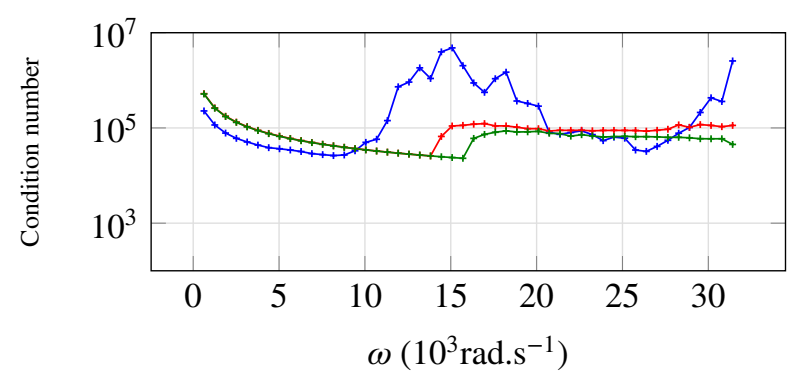

(d) P4

FIGURE 9 Maximal condition number (infinity norm) of the matrix $\mathbb{A}^{K}$ as a function of the frequency, for three materials and four interpolation orders. The characteristics of the media are presented in table 1 . The four stabilization parameters are set to 1. The results for sand are represented in blue $\square$, for shale in red $\square$ and for sandstone in green $\square$ 
- We now move on to the global system $\mathbb{K} \underline{\Lambda}=\mathbb{S}$. As previously done, we address the question of well-posedness numerically by computing the condition number of the global matrix $\mathbb{K}$ with the hope it is not too high, which ensures that the matrix can be inverted. As formerly, we consider three different media which are composed of sand, shale or sandstone (see Table 11. The condition number of $\mathbb{K}$ is displayed in Figure 7 as a function of the stabilization parameters. The condition number for the test with sand is lower than the one for shale and sandstone. Moreover, the curves have similar trends for every interpolation order. In the interval in consideration for the stabilization parameters, the condition number is stable. This means that the accuracy of the resolution of the global system is not sensitive to the value of the stabilization parameters.

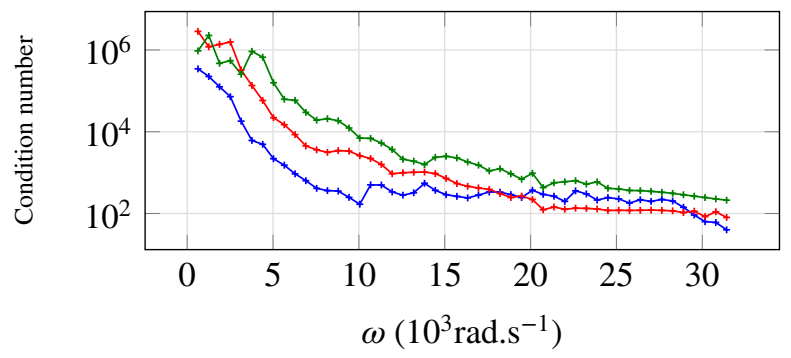

(a) P1

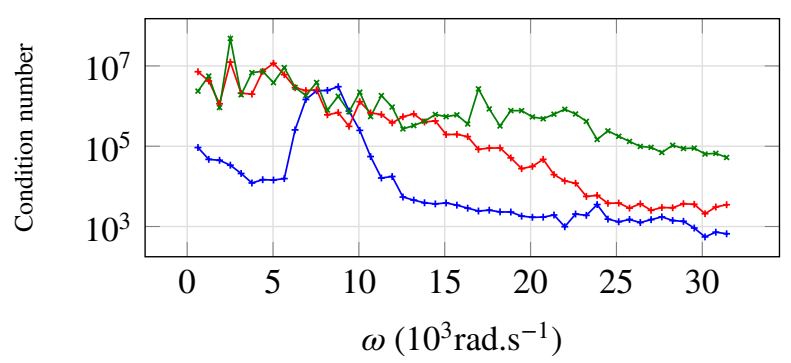

(c) P3

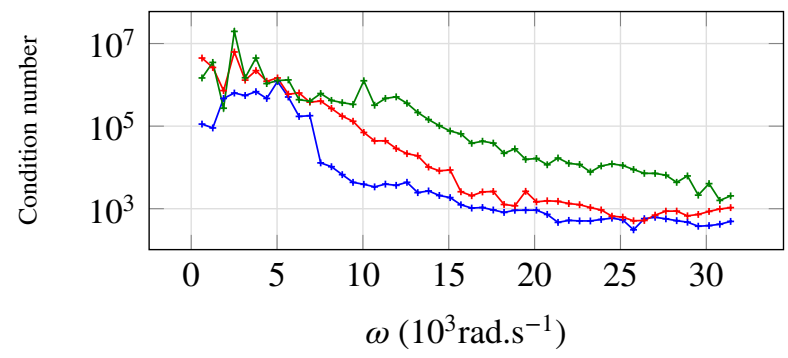

(b) P2

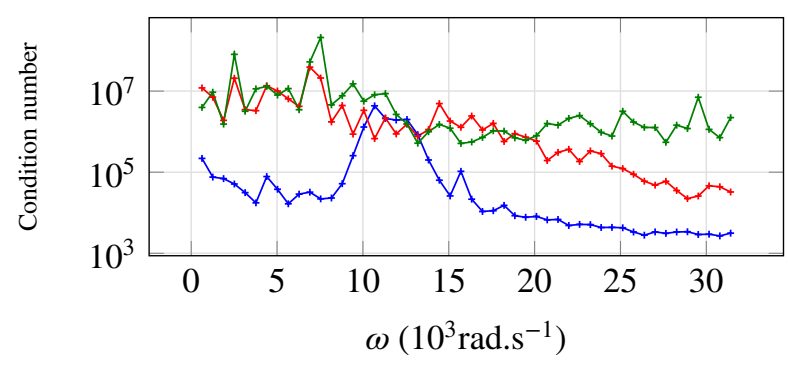

(d) P4

FIGURE 10 Condition number (infinity-norm) of the global matrix $\mathbb{K}$ as a function of the frequency, for three materials and four interpolation orders. The characteristics of the media are presented in table 1 All stabilization parameters are set to 1 . The results for sand are represented in blue $\square$ for shale in red $\square$ and for sandstone in green $\square \square$

Influence of the frequency We continue the numerical study of well-posedness by analyzing the influence of the frequency on the condition number of the linear system. Note that the parameters change with frequency. As previously done, we first consider the local system and then the global one.

Remark 4. The goal of this experiment is not to analyze precisely the variations of the condition number, but to show that the condition number is lower than $10^{8}$ for each triangle, for the three types of media and for the considered range of frequency, in order to show that all local systems are invertible. Indeed, it is acknowledged that the inversion can be made without difficulties if the condition number is lower than $10^{16}$.

- First, for a domain composed of shale, we consider an interior element of the mesh. We denote by $h$ the diameter of the element and by $\lambda_{\mathrm{B}}$ the wavelength corresponding to the slow wave, and we display the condition number of the elementary matrix $\mathbb{A}^{K}$ as a function of $\frac{h}{\lambda_{\mathrm{B}}}$, the number of wavelengths in the element. The results are given in Figure 8 We observe that the condition number increases with the interpolation orders, but stays however bounded below $10^{6}$. Then, for the three different media described in Table 1 , we show the maximal condition number of the matrices $\mathbb{A}^{K}$ on each element as a function of the frequency in Figure 9 . For sand, the curves are less smooth than for sandstone and shale. We observe here that there is no conflict between the accuracy and the condition number.

- Finally, the condition number of the global matrix $\mathbb{K}$ is presented in Figure 10 as a function of the frequency. We observe that the condition number of the elementary matrix decreases with the frequency. 
As a conclusion, the condition number does not increase with the accuracy (or the number of points per wavelength), and we can obtain both a well conditioned system and an accurate solution.

\section{3 | Layered domain}

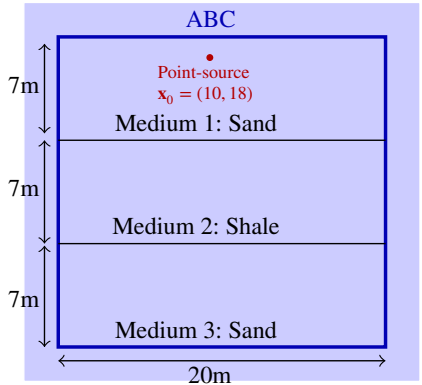

(a) Domain

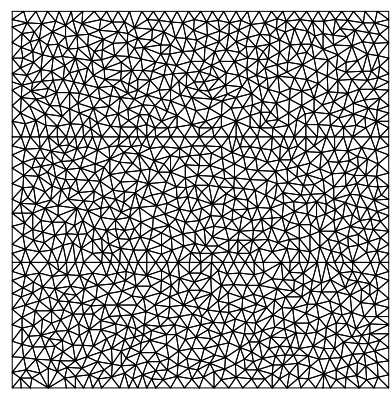

(b) Mesh

FIGURE 11 Domain and mesh used for the three layers test. The parameters of the three media are detailed in Table 1

In this section we apply the HDG method to a stratified geophysical medium composed of three horizontal layers made up of different materials. The domain and the mesh used for this test are shown in Figure 11 On the boundary of the domain, we impose an outgoing radiation condition which was constructed in ${ }^{48}$, equation 38 . The velocities and wavelengths $\lambda=\frac{v}{f}$, of the three types of waves in the sand and the shale are given in Table 5 for frequency $f=500 \mathrm{~Hz}$.

\begin{tabular}{|l|c|c|}
\hline Physical parameters & Sand & Shale \\
\hline $\mathrm{v}_{\mathrm{P}}\left(\mathrm{m} \cdot \mathrm{s}^{-1}\right)$ & $\left(1.8810^{3}, 10.4\right)$ & $2.4810^{3}$ \\
$\mathrm{v}_{\mathrm{S}}\left(\mathrm{m} \cdot \mathrm{s}^{-1}\right)$ & $\left(4.9310^{2}, 3.76\right)$ & $1.4310^{3}$ \\
$\mathrm{v}_{\mathrm{B}}\left(\mathrm{m} . \mathrm{s}^{-1}\right)$ & $\left(2.5710^{2}, 57.9\right)$ & $1.1310^{3}$ \\
\hline$\lambda_{\mathrm{P}}(\mathrm{m})$ & 3.76 & 4.96 \\
$\lambda_{\mathrm{S}}(\mathrm{m})$ & 0.987 & 2.86 \\
$\lambda_{\mathrm{B}}(\mathrm{m})$ & 0.562 & 2.03 \\
\hline
\end{tabular}

TABLE 5 Velocities and wavelengths for $f=500 \mathrm{~Hz}$ in the materials in consideration for the test described in 11

Regarding the external source, we consider a point-source at position $\mathbf{x}_{0} \in \mathbb{R}^{2}$, modeled by Dirac-type distributions $\mathcal{E}^{\prime}$ acting on smooth vector-valued test functions in $\mathcal{E}:=\left(C^{\infty}\right)^{2}$. In (1), we first consider the problem with $\mathbf{f}_{u}=\nabla \delta_{0}\left(\mathbf{x}-\mathbf{x}_{0}\right)$, which corresponds to a source in longitudinal waves $(\mathrm{P}, \mathrm{B})$. We then consider the problem with $\mathbf{f}_{u}=\nabla \times \delta\left(\mathbf{x}-\mathbf{x}_{0}\right)$ to have a source in transverse wave $\mathrm{S}$. The action of these distributions are defined, for $\mathbf{v}=\left(v_{x}, v_{y}\right)^{t} \in \mathcal{E}$,

$$
\left\langle\nabla \delta\left(\mathbf{x}-\mathbf{x}_{0}\right), \mathbf{v}\right\rangle_{\mathcal{E}^{\prime}, \mathcal{E}}=-(\nabla \cdot \mathbf{v})\left(\mathbf{x}_{0}\right) \quad, \quad\left\langle\nabla \times \delta\left(\mathbf{x}-\mathbf{x}_{0}\right), \mathbf{v}\right\rangle_{\mathcal{E}^{\prime}, \mathcal{E}}=-\left(\partial_{x} v_{y}-\partial_{y} v_{x}\right)\left(\mathbf{x}_{0}\right) .
$$

The results due to the point sources in (58) using an interpolation order 6 are given in Figure 12 For the case of longitudinal waves, in Figure 12(a) and 12(b), we observe both B and P waves in the upper layer around the source. On the solid velocity, we observe mainly the $\mathrm{P}$ wave in layers 2 and 3 , with the B wave mostly absorbed. However, we can observe the presence of the B wave on the relative fluid velocity in the middle layer. For the case with a source in transverse wave in Figure 14(b), we mainly observe the $\mathrm{S}$ wave in the three layers. From these initial observations, we use the following procedure to separate and highlight 


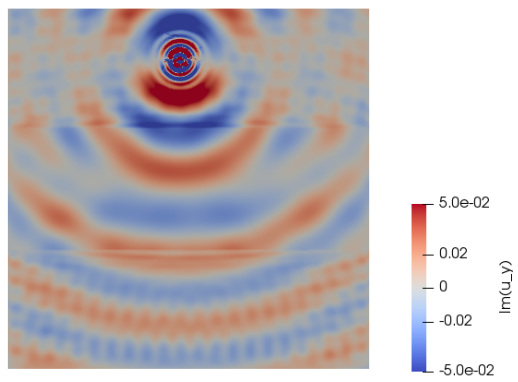

(a) $\mathrm{u}_{y}$ with $\mathbf{f}_{u}=\nabla\left(\delta_{0}\left(\mathbf{x}-\mathbf{x}_{0}\right)\right)$

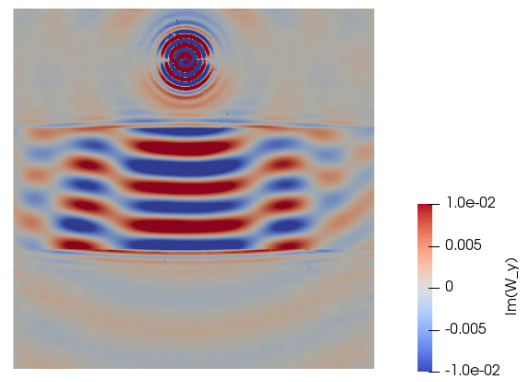

(b) $\mathrm{w}_{y}$ with $\mathbf{f}_{u}=\nabla\left(\delta_{0}\left(\mathbf{x}-\mathbf{x}_{0}\right)\right)$

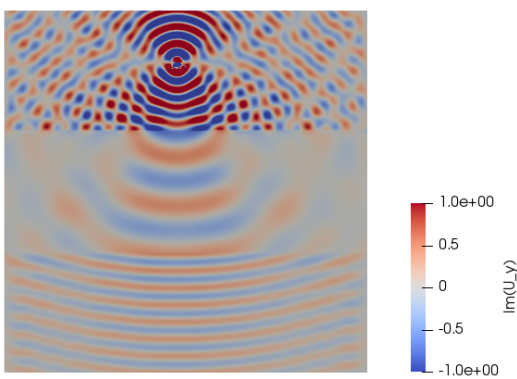

(c) $\mathrm{u}_{y}$ with $\mathbf{f}_{u}=\nabla \times\left(\delta_{0}\left(\mathbf{x}-\mathbf{x}_{0}\right)\right)$

FIGURE 12 Vertical component of solid velocity $\mathrm{u}_{y}$ and relative fluid velocity $\mathrm{w}_{y}$ on three layers domain for two types of point source at frequency $f=500 \mathrm{~Hz}$. For Figures (a) and (b), in the lowest layer, we observe 2 wavelengths in a vertical space of 7 meters, which means that the wavelength $\lambda$ is approximately $3.5 \mathrm{~m}$ and agrees with that of $\mathrm{P}$ wave, cf. Table 5 . In Figure (c), the lowest layer contains 7 wavelengths within a depth of $7 \mathrm{~m}$, which means that $\lambda$ is approximately $1 \mathrm{~m}$, and thus corresponds to that of $\mathrm{S}$ wave, cf. Table 5 The resolution of the global system takes 6.90 seconds (CPU time), and it needs $659 \mathrm{MB}$ of memory to solve the global system.

the three waves. In order to show the transverse wave, we display in Figure 13(c) and 14(c) the curl of the solid velocity,

$$
\mathrm{W}_{\mathrm{S}}:=\operatorname{curl} \mathbf{u}=\partial_{x} \mathrm{u}_{y}-\partial_{y} \mathrm{u}_{x} .
$$

Regarding the longitudinal waves, in order to eliminate the $\mathrm{B}$ wave and keep the $\mathrm{P}$ wave, we compute the quantity

$$
\mathrm{W}_{\mathrm{P}}:=\nabla \cdot \mathbf{w}-\beta_{\mathrm{B}} \nabla \cdot \mathbf{u} .
$$

This is shown in Figure 13(a) and 14(a). Similarly, to keep the B wave and eliminate the P one, in Figure 13(b) and 14(b), we compute

$$
\mathrm{W}_{\mathrm{B}}:=\nabla \cdot \mathbf{w}-\beta_{\mathrm{P}} \nabla \cdot \mathbf{u} .
$$

As expected, Figure 13(a) illustrates the fact that the longitudinal source generates mostly $\mathrm{P}$ and B waves with a small $\mathrm{S}$ waves

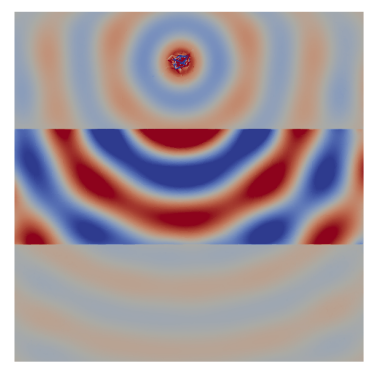

(a) $\mathrm{P}$ wave $\mathrm{W}_{\mathrm{P}}$ defined in 60

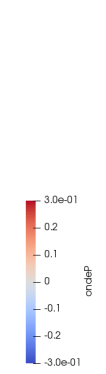

60

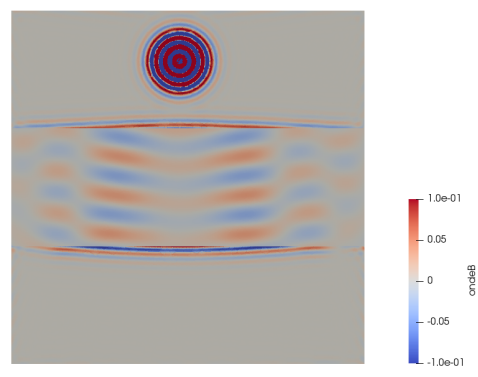

(b) $\mathrm{B}$ wave $\mathrm{W}_{\mathrm{B}}$ defined in 61

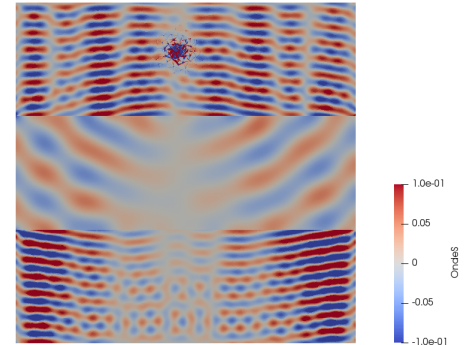

(c) $\mathrm{S}$ wave $\mathrm{W}_{\mathrm{S}}$ defined in 59

FIGURE 13 Illustration of the three waves for the source $\mathbf{f}_{u}=\nabla \delta_{0}\left(\mathbf{x}-\mathbf{x}_{0}\right)$ at frequency $f=500 \mathrm{~Hz}$. In Fig. 13(b), B-wave $\mathrm{W}_{\mathrm{B}}$ is seen around the source and but is mostly absorbed in the first layer. We see its reflection on the interfaces. The B-wave propagates in the second layer because there is no absorption, but is absorbed in the third layer. For the S-wave, we observe an artifact at the position of the source which contains only the compression waves.

artifact. The attenuation acts mostly on the B wave, which is damped before reaching the first interface. On the contrary, the $\mathrm{P}$ wave is not damped and generates transmitted P, B and S waves in the second layer and reflected waves in the first layer. The reflected $\mathrm{P}$ wave is barely visible, hidden by the incident $\mathrm{P}$ wave, while the reflected $\mathrm{B}$ wave is almost immediately damped. Only the reflected $\mathrm{S}$ wave is visible. The three transmitted waves propagate without attenuation in the second layer and generate 


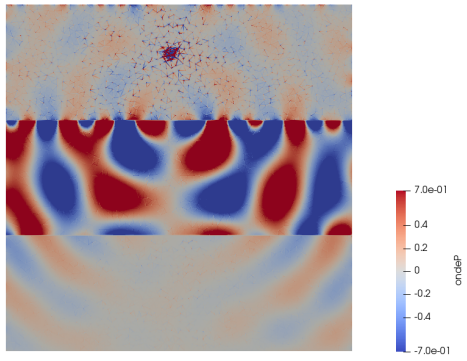

(a) $\mathrm{P}$ wave $\mathrm{W}_{\mathrm{P}}$ defined in 60

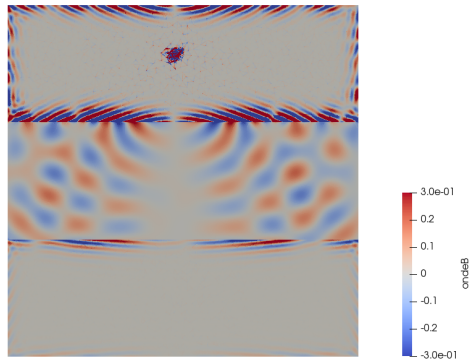

(b) $\mathrm{B}$ wave $\mathrm{W}_{\mathrm{B}}$ defined in 61

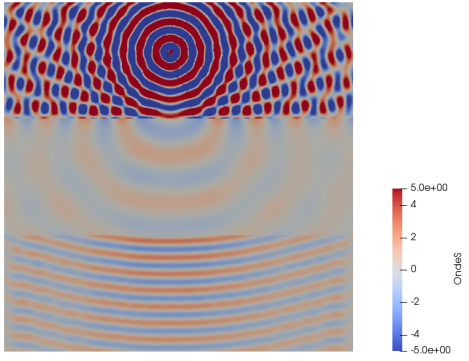

(c) $\mathrm{S}$ wave $\mathrm{W}_{\mathrm{S}}$ defined in 59

FIGURE 14 Illustration of the three waves with $\mathrm{W}_{\mathrm{P}}, \mathrm{W}_{\mathrm{B}}$, and $\mathrm{W}_{\mathrm{S}}$, defined in (60), (61), and (59) respectively, for the source $\mathbf{f}_{u}=\nabla \times\left(\delta\left(\mathbf{x}-\mathbf{x}_{0}\right)\right)$ which corresponds to a source in transverse wave. The solution is given for frequency $f=500 \mathrm{~Hz}$. As the source is a S-wave, the longitudinal waves are not around the source and we observe an artifact. However, at the interfaces, with the reflections, $\mathrm{P}$ and B-waves appear and propagate in the material. The S-wave is reflected on the boundary of the first layer which is converted into compression waves. In the second layer, the B-wave propagates and is not absorbed. The $\mathrm{S}$ wave propagates correctly around the source and in the three layers.

transmitted waves in the third layer. Once again, the transmitted B wave is damped while the $\mathrm{S}$ and $\mathrm{B}$ waves propagate until the boundary of the domain. Note the efficiency of the absorbing boundary conditions for this test case. In fig. 14(b) we see that, as expected, the source produces almost only $\mathrm{S}$ waves which generate mostly transmitted $\mathrm{P}$ and $\mathrm{B}$ waves at the interface with the second layer and reflected B waves at the top boundary. These latter decay rapidly, due to the attenuation.

\section{Comparison with anisotropic material:}

Here, we still consider the stratified geophysical medium composed of three horizontal layers of different materials. However, we modify the composition of the upper layer which is now anisotropic sand, whose characteristic parameters are given in Table

1. On the boundary of the computational domain, since the radiation condition in ${ }^{48}$ was obtained under the assumption that the medium is isotropic, we truncate the domain with a PML. The corresponding domain is shown in Figure $15(\mathrm{a})$ The results of this experiment are given in Figure 15(c) and can be compared with Figure 15(b) which corresponds to the case where the upper layer is filled with isotropic sand.

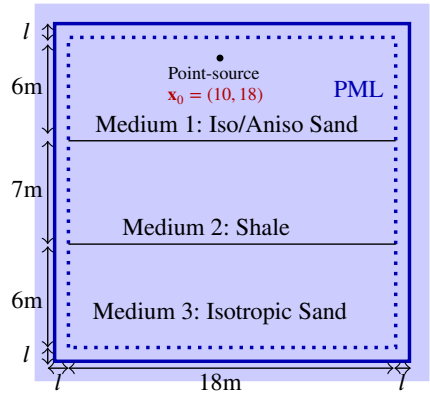

(a) Computational domain with $l=1 \mathrm{~m}$

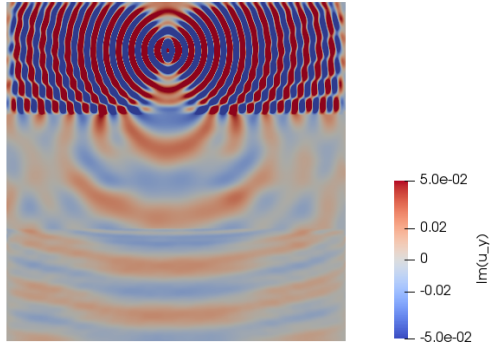

(b) $\mathrm{u}_{y}$ with isotropic sand

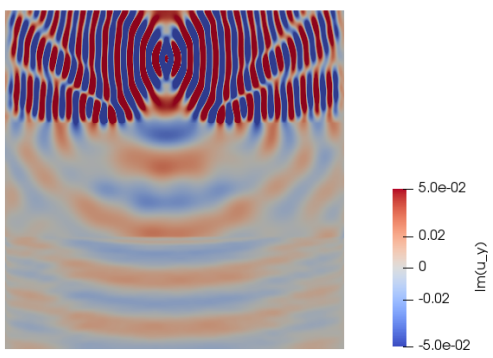

(c) $\mathrm{u}_{y}$ with anisotropic sand

FIGURE 15 Vertical component of solid velocity $\mathrm{u}_{y}$ in a three layered domain with a point source $\mathbf{f}_{u}=\left(\delta_{0}\left(\mathbf{x}-\mathbf{x}_{0}\right)\right) \mathbf{e}_{y}$ at frequency $f=500 \mathrm{~Hz}$, for two different kind of media in the top layer.

In figure 15(c) we observe that in the upper layer, the wavefront has an elliptical shape which is characteristic of an anisotropic phenomenon, whereas the wavefront is indeed circular in figure 15(b) The shape of the wavefront, more stretched in the vertical direction, indicates that the velocity of the wave is greater in this direction than in the other. This observation is consistent with 
the fact that the number of wavelengths propagated in the horizontal direction is greater in anisotropic sand than in isotropic sand. This experiment also shows that the simulation remains stable with the introduction of anisotropy. Moreover, PML does not seem to generate more reflections in the anisotropic medium than in the isotropic medium. It can also be observed that the signal in the deepest layer of Figure 15(c) is very close to that of Figure $15(\mathrm{~b})$ The difference is more noticeable in the second layer but not very important.

\section{6 | CONCLUSION}

In this work, we have developed and implemented a HDG method to simulate wave propagation in 2D anisotropic poroelastic media, governed by Biot's model. We have worked in frequency domain, in order to handle the frequency-dependent parameters which are pseudo-differential operators in the time domain. We provided a detailed description of the numerical algorithm and the different steps required for its development. Our HDG method was validated by comparing with reference solutions constructed in a previous work ${ }^{38}$. In our numerical investigation on the influence of stabilization parameters on the convergence of the method, we have shown that the four stabilization parameters are necessary for the method to keep an optimal order of convergence $(p+1)$. We also note that the strain tensor's symmetry was imposed strongly, which ensures that we do not lose half an order of convergence. We have also studied numerically the condition number of the global and local problems, and show they remain in a good range that maintains stability for the geophysical material in consideration. The materials we have chosen are typical materials found in geophysical experiments. Hence, by taken the input parameters of magnitude given in section 2.2 , the normalized values of coefficients given in Tab. 1] and the four penalization parameters of same value in the range of $\left[10^{-4}, 10^{4}\right]$, our work offers immediate utility for simulating the subsurfaces of the Earth. Finally, we illustrate the three types of poroelastic waves in a 3-layer media. This work lays the foundation for future investigation such as improvement of convergence order to $\mathrm{p}+2$ by using a post-processing method, and construction of absorbing boundary conditions for simulation in infinite domains ${ }^{48}$.

How to cite this article: Barucq H., Diaz J., Meyer R., and Pham H. (2020), Implementation of HDG method for time-harmonic anisotropic poroelastic in two dimensions., ..., ....

\section{APPENDIX}

\section{A ELEMENTARY MATRICES}

In section 4 , we obtained from the discretization of (16) the system $\mathbb{A}^{K} \underline{W}^{K}+\mathbb{B}^{K} \underline{\Lambda}^{K}=\mathbb{C}_{\text {source }}^{K}$, where $\underline{W}^{K}$ and $\underline{\Lambda}^{K}$ are defined

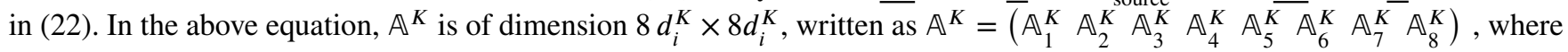

$$
\mathbb{A}_{1}^{K}=\left(\begin{array}{c}
\mathrm{i} \omega \rho_{a}^{K} \mathbb{M}^{K}+\sum_{f=1}^{3} \gamma_{1} \mathbb{E}^{\beta(K, f)} \\
0 \\
i \omega \rho_{f}^{K} \mathbb{M}^{K} \\
0 \\
\left(C_{11}^{K} \mathbb{D}_{x}^{K}+C_{13}^{K} \mathbb{D}_{y}^{K}\right) \\
\left(C_{12}^{K} \mathbb{D}_{x}^{K}+C_{23}^{K} \mathbb{D}_{y}^{K}\right) \\
\left(C_{13}^{K} \mathbb{D}_{x}^{K}+C_{33}^{K} \mathbb{D}_{y}^{K}\right) \\
-M^{K}\left(\sum_{f=1}^{3} \gamma_{4} \mathbb{J}_{x}^{\beta(K, f)}+\alpha_{11}^{K} \mathbb{D}_{x}^{K}+\alpha_{12}^{K} \mathbb{D}_{y}^{K}\right)
\end{array}\right), \quad \mathbb{A}_{2}^{K}=\left(\begin{array}{c}
0 \\
\mathrm{i} \omega \rho_{a}^{K} \mathbb{M}^{K}+\sum_{f=1}^{3} \gamma_{1} \mathbb{E}^{\beta(K, f)} \\
0 \\
\mathrm{i} \omega \rho_{f}^{K} \mathbb{M}^{K} \\
\left(C_{13}^{K} \mathbb{D}_{x}^{K}+C_{12}^{K} \mathbb{D}_{y}^{K}\right) \\
\left(C_{23}^{K} \mathbb{D}_{x}^{K}+C_{22}^{K} \mathbb{D}_{y}^{K}\right) \\
\left(C_{33}^{K} \mathbb{D}_{x}^{K}+C_{23}^{K} \mathbb{D}_{y}^{K}\right) \\
-M^{K}\left(\sum_{f=1}^{3} \gamma_{4} \mathbb{D}_{y}^{\beta(K, f)}+\alpha_{12}^{K} \mathbb{D}_{x}^{K}+\alpha_{22}^{K} \mathbb{D}_{y}^{K}\right)
\end{array}\right) \text {, }
$$




$$
\begin{aligned}
& \mathbb{A}_{3}^{K}=\left(\begin{array}{c}
\mathrm{i} \omega \rho_{f}^{K} \mathbb{M}^{K} \\
0 \\
\mathrm{i} \omega \rho_{\mathrm{dyn}}^{K} \mathbb{M}^{K} \\
0 \\
0 \\
0 \\
0 \\
M^{K}\left(\mathbb{D}_{x}^{K}\right)^{T}
\end{array}\right), \quad \mathbb{A}_{4}^{K}=\left(\begin{array}{c}
0 \\
\mathrm{i} \omega \rho_{f}^{K} \mathbb{M}^{K} \\
0 \\
\mathrm{i} \omega \rho_{\mathrm{dyn}}^{K} \mathbb{M}^{K} \\
0 \\
0 \\
0 \\
M^{K}\left(\mathbb{D}_{y}^{K}\right)^{T}
\end{array}\right) \quad \mathbb{A}_{5}^{K}=\left(\begin{array}{c}
-\left(\mathbb{D}_{x}^{K}\right)^{T} \\
0 \\
0 \\
0 \\
\mathrm{i} \omega \mathbb{M}^{K} \\
0 \\
0 \\
0
\end{array}\right), \quad \mathbb{A}_{6}^{K}=\left(\begin{array}{c}
0 \\
-\left(\mathbb{D}_{y}^{K}\right)^{T} \\
0 \\
0 \\
0 \\
\mathrm{i} \omega \mathbb{M}^{K} \\
0 \\
0
\end{array}\right), \quad \mathbb{A}_{7}^{K}=\left(\begin{array}{c}
-\left(\mathbb{D}_{y}^{K}\right)^{T} \\
-\left(\mathbb{D}_{x}^{K}\right)^{T} \\
0 \\
0 \\
0 \\
0 \\
\mathrm{i} \omega \mathbb{M}^{K} \\
0
\end{array}\right) \\
& \mathbb{A}_{8}^{K}=\left(\sum_{f=1}^{3} \gamma_{3} \rrbracket_{x}^{\beta(K, f)}, \sum_{f=1}^{3} \gamma_{3} \rrbracket_{y}^{\beta(K, f)},-\mathbb{D}_{x}^{K},-\mathbb{D}_{y}^{K}, \mathrm{i} \omega \alpha_{11}^{K} \mathbb{M}^{K}, \mathrm{i} \omega \alpha_{22}^{K} \mathbb{M}^{K}, \mathrm{i} \omega \alpha_{12}^{K} \mathbb{M}^{K}, \mathrm{i} \omega \mathbb{M}^{K}-\sum_{f=1}^{3} M \gamma_{2} \mathbb{E}^{\beta(K, f)}\right)^{T} .
\end{aligned}
$$

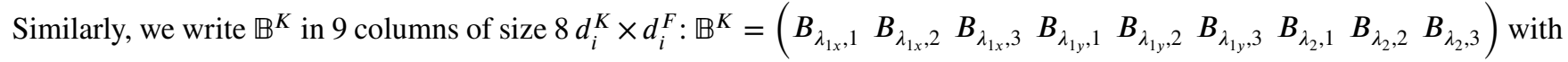

$$
B_{\lambda_{1 x}, f}=\left(\begin{array}{c}
-\gamma_{1} \mathbb{F}^{\beta(K, f)} \\
0 \\
0 \\
0 \\
\left(-C_{11}^{K} \mathbb{Q}_{x}^{\beta(K, f)}-C_{13}^{K} \mathbb{Q}_{y}^{\beta(K, f)}\right) \\
\left(-C_{12}^{K} \mathbb{Q}_{x}^{\beta(K, f)}-C_{23}^{K} \mathbb{Q}_{y}^{\beta(K, f)}\right) \\
\left(-C_{13}^{K} \mathbb{Q}_{x}^{\beta(K, f)}-C_{33}^{K} \mathbb{Q}_{y}^{\beta(K, f)}\right) \\
M^{K}\left(\left(\gamma_{4}+\alpha_{11}^{K}\right) \mathbb{Q}_{x}^{\beta(K, f)}+\alpha_{12}^{K} \mathbb{Q}_{y}^{\beta(K, f)}\right)
\end{array}\right), \quad B_{\lambda_{1 y}, f}=\left(\begin{array}{c}
0 \\
-\gamma_{1} \mathbb{F}^{\beta(K, f)} \\
0 \\
0 \\
\left(-C_{13}^{K} \mathbb{Q}_{x}^{\beta(K, f)}-C_{12}^{K} \mathbb{Q}_{y}^{\beta(K, f)}\right) \\
\left(-C_{23}^{K} \mathbb{Q}_{x}^{\beta(K, f)}-C_{22}^{K} \mathbb{Q}_{y}^{\beta(K, f)}\right) \\
\left(-C_{33}^{K} \mathbb{Q}_{x}^{\beta(K, f)}-C_{23}^{K} \mathbb{Q}_{y}^{\beta(K, f)}\right) \\
M^{K}\left(\alpha_{12}^{K} \mathbb{Q}_{x}^{\beta(K, f)}+\left(\gamma_{4}+\alpha_{22}\right) \mathbb{Q}_{y}^{\beta(K, f)}\right)
\end{array}\right), \quad B_{\lambda_{2}, f}=\left(\begin{array}{c}
-\gamma_{3} \mathbb{Q}_{x}^{\beta(K, f)} \\
-\gamma_{3} \mathbb{Q}_{y}^{\beta(K, f)} \\
\mathbb{Q}_{x}^{\beta(K, f)} \\
\mathbb{Q}_{y}^{\beta(K, f)} \\
0 \\
0 \\
0 \\
M^{K} \gamma_{2} \mathbb{F}^{\beta(K, f)}
\end{array}\right),
$$

for $f=1,2,3$.

The matrices $\mathbb{P}^{K}$ and $\mathbb{T}^{K}$ are:

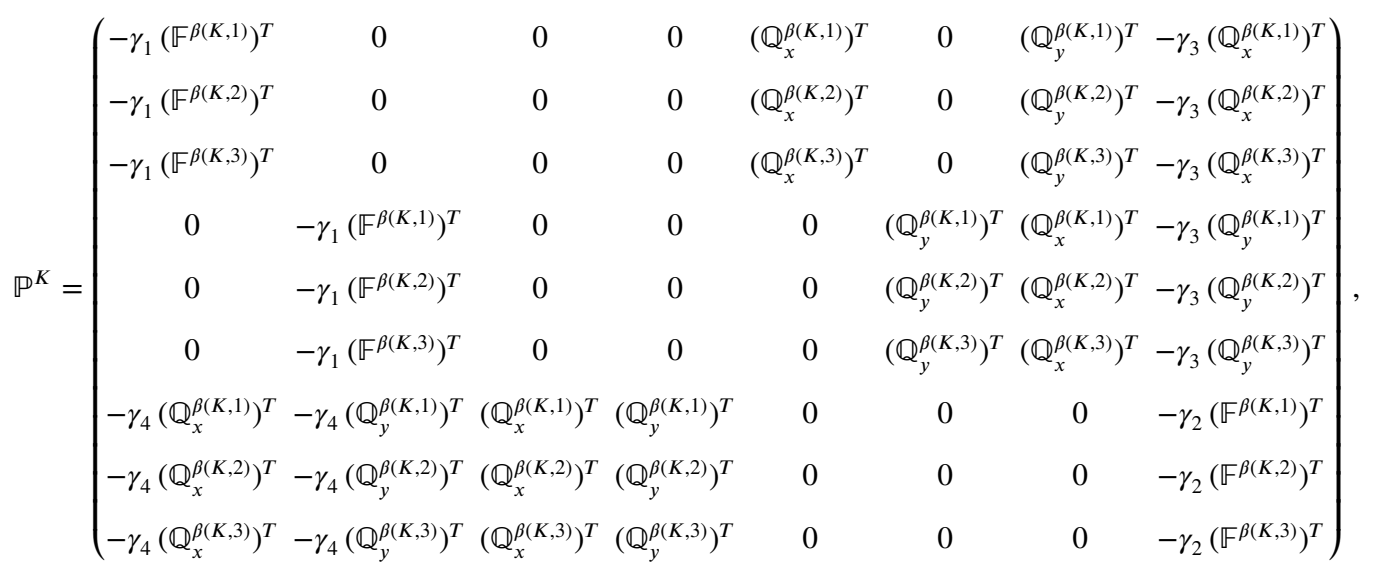

and

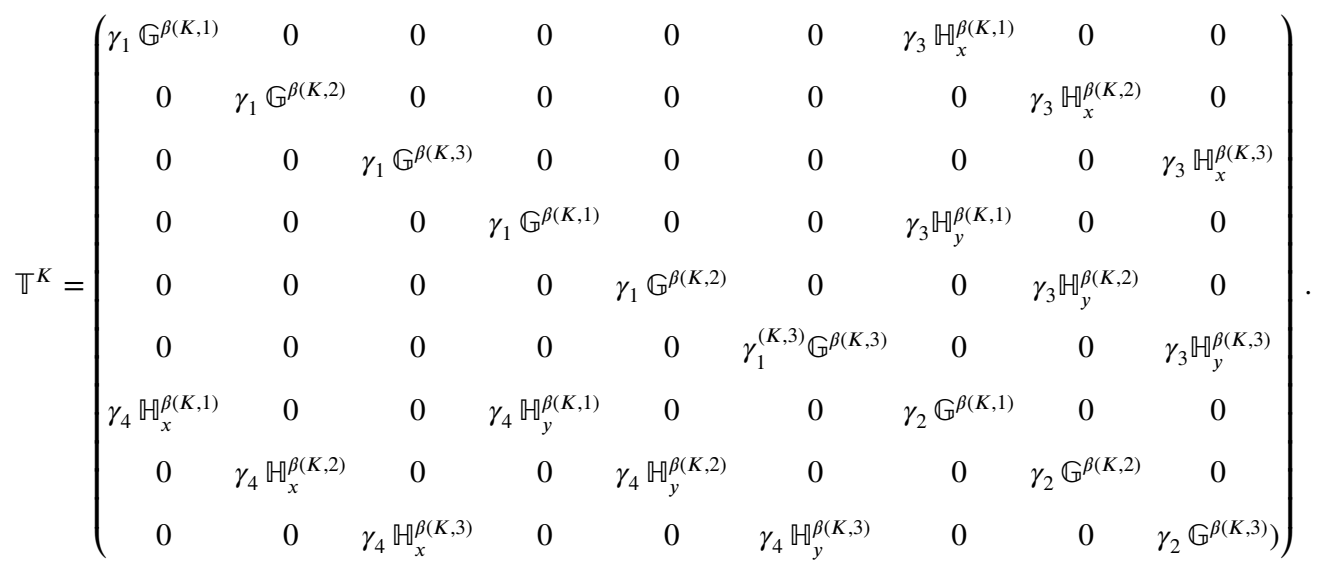




\section{B DETAILS FOR THE IMPLEMENTATION OF BOUNDARY CONDITIONS OF TYPE 4}

For a face on the boundary of the domain, we can impose four types of boundary conditions, see section 2.3 . In the case of type 1 , we impose the continuity of $\boldsymbol{\tau}_{h} \boldsymbol{n}$ and $\mathbf{w} \cdot \boldsymbol{n}$, see equation (4a). This means that the transmission conditions (39) for an interior interface and on the boundary of the mesh are the same. In the following, we detail the expression of the elementary matrices $\mathbb{P}^{K}$ and $\mathbb{T}^{K}$ in the case where we impose the continuity of $\mathbf{u}$ and $\mathrm{p}$, as in equation (4c). The two other formulations for the boundary conditions are linear combinations of these two formulations. From equation $\sqrt{4 \mathrm{c}}$, we impose:

$$
\left\{\begin{array} { l } 
{ \mathbf { u } _ { h } = f _ { u } , } \\
{ \mathrm { p } _ { h } = f _ { p } , }
\end{array} \quad \Rightarrow \quad \left\{\begin{array}{l}
\underline{\lambda}_{1}=f_{u}, \\
\underline{\lambda}_{2}=f_{p},
\end{array}\right.\right.
$$

with $f_{u}$ and $f_{p}$ some exterior forces. Let us consider an element $K$ with the face corresponding to the face 1 in local numbering on the boundary of the domain $\Omega$. In this case, the elementary matrices become:

$$
\mathbb{P}^{K}=\left(\begin{array}{cccccccc}
0 & 0 & 0 & 0 & 0 & 0 & 0 & 0 \\
-\gamma_{1}\left(\mathbb{F}^{\beta(K, 2)}\right)^{T} & 0 & 0 & 0 & \left(\mathbb{Q}_{x}^{\beta(K, 2)}\right)^{T} & 0 & \left(\mathbb{Q}_{y}^{\beta(K, 2)}\right)^{T} & -\gamma_{3}\left(\mathbb{Q}_{x}^{\beta(K, 2)}\right)^{T} \\
-\gamma_{1}\left(\mathbb{F}^{\beta(K, 3)}\right)^{T} & 0 & 0 & 0 & \left(\mathbb{Q}_{x}^{\beta(K, 3)}\right)^{T} & 0 & \left(\mathbb{Q}_{y}^{\beta(K, 3)}\right)^{T} & -\gamma_{3}\left(\mathbb{Q}_{x}^{\beta(K, 3)}\right)^{T} \\
0 & 0 & 0 & 0 & 0 & 0 & 0 & 0 \\
0 & -\gamma_{1}\left(\mathbb{F}^{\beta(K, 2)}\right)^{T} & 0 & 0 & 0 & \left(\mathbb{Q}_{y}^{\beta(K, 2)}\right)^{T} & \left(\mathbb{Q}_{x}^{\beta(K, 2)}\right)^{T} & -\gamma_{3}\left(\mathbb{Q}_{y}^{\beta(K, 2)}\right)^{T} \\
0 & -\gamma_{1}\left(\mathbb{F}^{\beta(K, 3)}\right)^{T} & 0 & 0 & 0 & \left(\mathbb{Q}_{y}^{\beta(K, 3)}\right)^{T} & \left(\mathbb{Q}_{x}^{\beta(K, 3)}\right)^{T} & -\gamma_{3}\left(\mathbb{Q}_{y}^{\beta(K, 3)}\right)^{T} \\
0 & 0 & 0 & 0 & 0 & 0 & 0 & 0 \\
-\gamma_{4}\left(\mathbb{Q}_{x}^{\beta(K, 2)}\right)^{T} & -\gamma_{4}\left(\mathbb{Q}_{y}^{\beta(K, 2)}\right)^{T} & \left(\mathbb{Q}_{x}^{\beta(K, 2)}\right)^{T} & \left(\mathbb{Q}_{y}^{\beta(K, 2)}\right)^{T} & 0 & 0 & 0 & -\gamma_{2}\left(\mathbb{F}^{\beta(K, 2)}\right)^{T} \\
-\gamma_{4}\left(\mathbb{Q}_{x}^{\beta(K, 3)}\right)^{T} & -\gamma_{4}\left(\mathbb{Q}_{y}^{\beta(K, 3)}\right)^{T} & \left(\mathbb{Q}_{x}^{\beta(K, 3)}\right)^{T} & \left(\mathbb{Q}_{y}^{\beta(K, 3)}\right)^{T} & 0 & 0 & 0 & -\gamma_{2}\left(\mathbb{F}^{\beta(K, 3)}\right)^{T}
\end{array}\right),
$$

and with $\llbracket$ denoting the identity matrix of size $d_{i}^{F} \times d_{i}^{F}$,

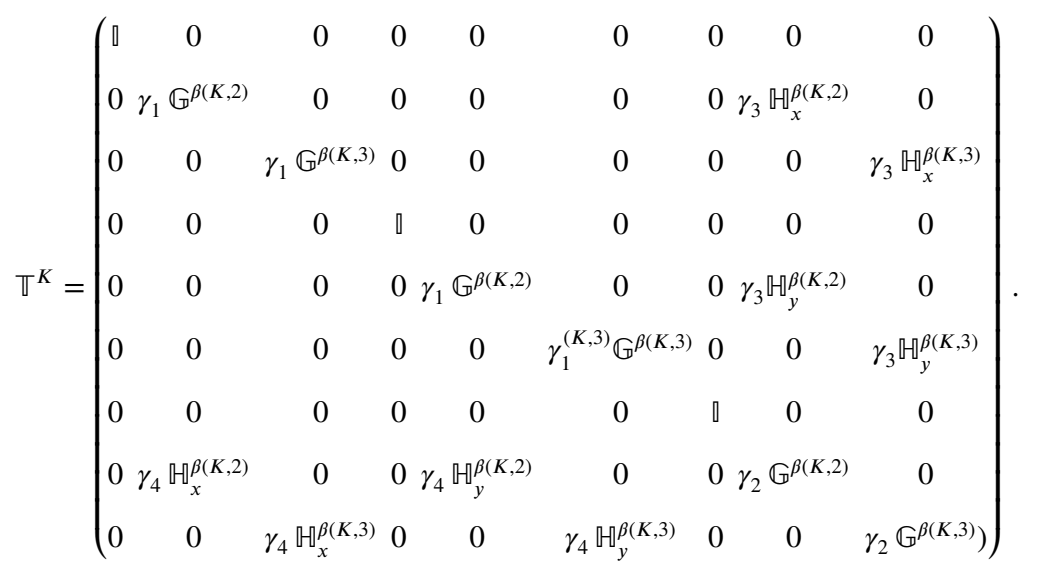

\section{ACKNOWLEDGEMENTS}

This project has received funding from the European Union's Horizon 2020 research and innovation program under the Marie Sklodowska-Curie grant agreement No 777778 (MATHROCKS).

The data that support the findings of this study are available from the corresponding author upon reasonable request. 


\section{References}

1. Anthony E, Vedanti N. Simulation of seismic wave propagation in poroelastic media using vectorized Biot's equations: an application to a CO2 sequestration monitoring case. Acta Geophysica 2020: 1-10.

2. Biot MA. General Theory of Three Dimensional Consolidation. Journal of Applied Physics 1941; 12(2): 155-164. doi: $10.1063 / 1.1712886$

3. Biot MA. Theory of Elasticity and Consolidation for a Porous Anisotropic Solid. Journal of Applied Physics 1955; 26(2): 182-185. doi: $10.1063 / 1.1721956$

4. Blanc E. Time-domain numerical modeling of poroelastic waves: the Biot-JKD model with fractional derivatives. Theses. Aix-Marseille Université, 2013.

5. Carcione JM, Santos JE, Picotti S. Anisotropic poroelasticity and wave-induced fluid flow: harmonic finite-element simulations. Geophysical Journal International 2011; 186(3): 1245-1254. doi: 10.1111/j.1365-246X.2011.05101.x

6. Lemoine GI, Ou MY, LeVeque RJ. High-resolution finite volume modeling of wave propagation in orthotropic poroelastic media. SIAM Journal on Scientific Computing 2013; 35(1): B176-B206. doi: 10.1137/120878720

7. Schanz M. Wave propagation in viscoelastic and poroelastic continua: a boundary element approach. Berlin; London: Springer . 2011.

8. Wenzlau F, Müller TM. Finite-difference modeling of wave propagation and diffusion in poroelastic media. GEOPHYSICS 2009; 74(4): T55-T66. doi: 10.1190/1.3122928

9. Serra Q, Ichchou M, Deü JF. Wave properties in poroelastic media using a Wave Finite Element Method. Journal of Sound and Vibration 2015; 335: 125-146. doi: 10.1016/j.jsv.2014.09.022

10. Ezziani A. Mathematical and Numerical modeling of Wave propagation in Viscoelastic and Poroelastic Media. Theses. ENSTA ParisTech, 2005.

11. Morency C, Tromp J. Spectral-element simulations of wave propagation in porous media. Geophysical Journal International 2008; 175(1): 301-345. doi: 10.1111/j.1365-246X.2008.03907.x

12. Dumbser M, Balsara DS, Toro EF, Munz CD. A unified framework for the construction of one-step finite volume and discontinuous Galerkin schemes on unstructured meshes. Journal of Computational Physics 2008; 227(18): 8209-8253. doi: $10.1016 /$ j.jcp.2008.05.025

13. de la Puente J, Dumbser M, Käser M, Igel H. Discontinuous Galerkin methods for wave propagation in poroelastic media. Geophysics 2008; 73(5): T77-T97. doi: 10.1190/1.2965027

14. Li X, Yao D, Lewis RW. A discontinuous Galerkin finite element method for dynamic and wave propagation problems in non-linear solids and saturated porous media. International Journal for Numerical Methods in Engineering 2003; 57(12): 1775-1800. doi: $10.1002 / \mathrm{nme} .741$

15. Shukla K, Hesthaven JS, Carcione JM, Ye R, de la Puente J, Jaiswal P. A nodal discontinuous Galerkin finite element method for the poroelastic wave equation. Computational Geosciences 2019; 23(3): 595-615. doi: 10.1007/s10596-019-9809-1

16. Dudley Ward N, Lähivaara T, Eveson S. A discontinuous Galerkin method for poroelastic wave propagation: The twodimensional case. Journal of Computational Physics 2017; 350: 690-727. doi: 10.1016/j.jcp.2017.08.070

17. Dazel O, Gabard G. Discontinuous Galerkin Methods for poroelastic materials. Proceedings of Meetings on Acoustics 2013; 19(1): 065004. doi: 10.1121/1.4799714

18. Dupuy B, De Barros L, Garambois S, Virieux J. Wave propagation in heterogeneous porous media formulated in the frequency-space domain using a discontinuous Galerkin method. GEOPHYSICS 2011; 76(4): N13-N28. doi: $10.1190 / 1.3581361$ 
19. Cockburn B. Static condensation, hybridization, and the devising of the HDG methods. In: Springer. 2016 (pp. 129-177).

20. Lozinski A. A primal discontinuous Galerkin method with static condensation on very general meshes. Numerische Mathematik 2019; 143(3): 583-604.

21. Giorgiani G, Fernández-Méndez S, Huerta A. Hybridizable discontinuous Galerkin p-adaptivity for wave propagation problems. International Journal for Numerical Methods in Fluids 2013; 72(12): 1244-1262.

22. Fernandez P, Christophe A, Terrana S, Nguyen NC, Peraire J. Hybridized discontinuous Galerkin methods for wave propagation. Journal of Scientific Computing 2018; 77(3): 1566-1604.

23. Cockburn B, Gopalakrishnan J, Lazarov R. Unified hybridization of discontinuous Galerkin, mixed, and continuous Galerkin methods for second order elliptic problems. SIAM Journal on Numerical Analysis 2009; 47(2): 1319-1365.

24. Soon SC, Cockburn B, Stolarski HK. A hybridizable discontinuous Galerkin method for linear elasticity. International Journal for Numerical Methods in Engineering 2009; 80(8): 1058-1092. doi: 10.1002/nme.2646

25. Cockburn B, Quenneville-Bélair V. Uniform-in-time superconvergence of the HDG methods for the acoustic wave equation. Mathematics of Computation 2014; 83(285): 65-85.

26. Nguyen NC, Peraire J, Cockburn B. High-order implicit hybridizable discontinuous Galerkin methods for acoustics and elastodynamics. Journal of Computational Physics 2011; 230(10): 3695-3718.

27. Li L, Lanteri S, Perrussel R. Numerical investigation of a high order hybridizable discontinuous Galerkin method for 2d time-harmonic Maxwell's equations. COMPEL - The international journal for computation and mathematics in electrical and electronic engineering 2013; 32(3): 1112-1138. doi: 10.1108/03321641311306196

28. Feng X, Lu P, Xu X. A hybridizable discontinuous Galerkin method for the time-harmonic Maxwell equations with high wave number. Computational Methods in Applied Mathematics 2016; 16(3): 429-445.

29. Bonnasse-Gahot M. High order discontinuous Galerkin methods for time-harmonic elastodynamics. Theses. Université Nice Sophia Antipolis, 2015.

30. Bonnasse-Gahot M, Calandra H, Diaz J, Lanteri S. Hybridizable discontinuous Galerkin method for the 2-D frequencydomain elastic wave equations. Geophysical Journal International 2018; 213(1): 637-659. doi: 10.1093/gji/ggx533

31. Griesmaier R, Monk P. Error analysis for a hybridizable discontinuous Galerkin method for the Helmholtz equation. Journal of Scientific Computing 2011; 49(3): 291-310.

32. Chaabane N, Rivière B. A splitting-based finite element method for the Biot poroelasticity system. Computers \& Mathematics with Applications 2018; 75(7): 2328-2337. doi: 10.1016/j.camwa.2017.12.009

33. Monforte L, Navas P, Carbonell JM, Arroyo M, Gens A. Low-order stabilized finite element for the full Biot formulation in soil mechanics at finite strain. International Journal for Numerical and Analytical Methods in Geomechanics 2019; 43(7): 1488-1515. doi: 10.1002/nag.2923

34. Kanschat G, Riviere B. A Finite Element Method with Strong Mass Conservation for Biot's Linear Consolidation Model. Journal of Scientific Computing 2018; 77(3): 1762-1779. doi: 10.1007/s10915-018-0843-2

35. Rodrigo C, Hu X, Ohm P, Adler J, Gaspar F, Zikatanov L. New stabilized discretizations for poroelasticity and the Stokes' equations. Computer Methods in Applied Mechanics and Engineering 2018; 341: 467-484. doi: 10.1016/j.cma.2018.07.003

36. Fu G. A high-order HDG method for the Biot's consolidation model. Computers \& Mathematics with Applications 2019; 77(1): 237-252. doi: 10.1016/j.camwa.2018.09.029

37. Hungria A. Using HDG+ to Compute Solutions of the 3D Linear Elastic and Poroelastic Wave Equations. PhD thesis. University of Delaware, 2019.

38. Barucq H, Diaz J, Meyer RC, Pham H. Analytic solutions and transmission eigenvalues in isotropic poroelasticity for bounded domain, scattering of obstacles and fluid-solid interaction problems in 2D. Research Report RR-9312, Inria; 2019. 
39. Carcione JM. Wave fields in real media: Wave propagation in anisotropic, anelastic, porous and electromagnetic media. Elsevier . 2007.

40. Kirby RM, Sherwin SJ, Cockburn B. To CG or to HDG:A Comparative Study. Journal of Scientific Computing 2012; 51(1): 183-212. doi: 10.1007/s10915-011-9501-7

41. Cockburn B, Gopalakrishnan J, Guzmàn J. A new elasticity element made for enforcing weak stress symmetry. Mathematics of Computation 2010; 79(271): 1331-1349. doi: 10.1090/S0025-5718-10-02343-4

42. Fu G, Cockburn B, Stolarski H. Analysis of an HDG method for linear elasticity. International Journal for Numerical Methods in Engineering 2015; 102(3-4): 551-575. doi: 10.1002/nme.4781

43. Griesmaier R, Monk P. Discretization of the wave equation using continuous elements in time and a hybridizable discontinuous Galerkin method in space. Journal of Scientific Computing 2014; 58(2): 472-498.

44. Barucq H, Diaz J, Meyer RC, Pham H. Implementation of HDG method for 2D anisotropic poroelastic first-order harmonic equations. Research Report RR-9326, Inria; 2020.

45. Garambois S, Dietrich M. Full waveform numerical simulations of seismoelectromagnetic wave conversions in fluidsaturated stratified porous media. Journal of Geophysical Research: Solid Earth 2002; 107(B7): ESE 5-1-ESE 5-18. doi: 10.1029/2001JB000316

46. Amestoy PR, Duff IS, L'Excellent JY, Koster J. A Fully Asynchronous Multifrontal Solver Using Distributed Dynamic Scheduling. SIAM Journal on Matrix Analysis and Applications 2001; 23(1): 15-41. doi: 10.1137/S0895479899358194

47. Anderson E. LAPACK users' guide. Philadelphia: Society for Industrial and Applied Mathematics . 1999.

48. Barucq H, Diaz J, Meyer RC, Pham H. Low-order absorbing boundary condition for two-dimensional isotropic poroelasticity. tech. rep., Inria; 2020.

49. Senior TBA, Volakis JL. Approximate boundary conditions in electromagnetics. London: Institution of Electrical Engineers . 1995. 